INSTITUT NATIONAL DE RECHERCHE EN INFORMATIQUE ET EN AUTOMATIQUE

\title{
Repartitors, selectors and superselectors
}

Frédéric Havet

$\mathbf{N}^{\circ} 5686$

Septembre 2005

Thème COM 



\title{
Repartitors, selectors and superselectors
}

\author{
Frédéric Havet* \\ Thème COM — Systèmes communicants \\ Projet Mascotte \\ Rapport de recherche $n^{\circ} 5686$ - Septembre 2005 - 28 pages
}

\begin{abstract}
An $(n, p, n+f)$-network $G$ is a graph $(V, E)$ where the vertex set $V$ is partitioned into four subsets $\mathcal{P}, \mathcal{I}, \mathcal{O}$ and $\mathcal{S}$ called respectively the priorities, the ordinary inputs, the outputs and the switches, satisfying the following constraints: there are $p$ priorities, $n-p$ ordinary inputs and $n+f$ outputs; each priority, each ordinary input and each output is connected to exactly one switch; switches have degree at most 4 . An $(n, p, n+f)$-network is a $(n, p, f)$-repartitor if for any disjoint subsets $\mathcal{F}$ and $\mathcal{B}$ of $\mathcal{O}$ with $|\mathcal{F}|=f$ and $|\mathcal{B}|=p$, there exist in $G, n$ edge-disjoint paths, $p$ of them from $\mathcal{P}$ to $\mathcal{B}$ and the $n-p$ others joining $\mathcal{I}$ to $\mathcal{O} \backslash(\mathcal{B} \cup \mathcal{F})$. The problem is to determine the minimum number $R(n, p, f)$ of switches of an $(n, p, f)$-repartitor and to construct a repartitor with the smallest number of switches.

In this paper, we show how to build general repartitors from $(n, 0, f)$-repartitors also called $(n, n+f)$-selectors. We then consrtuct selectors using more powerful networks called superselectors. An $(n, 0, n)$-network is an $n$-superselector if for any subsets $\mathcal{I}^{\prime} \subset \mathcal{I}$ and $\mathcal{O}^{\prime} \subset \mathcal{O}$ with $\left|\mathcal{O}^{\prime}\right|=\left|\mathcal{I}^{\prime}\right|$, there exist in $G,\left|\mathcal{O}^{\prime}\right|$ edge-disjoint paths joining $\mathcal{I}^{\prime}$ to $\mathcal{O}^{\prime}$. We show that the minimum number of switches of an $n$-superselector $S^{+}(n)$ is at most $17 n+O(\log (n))$. We then deduce that $R(n, p, f) \leq \frac{69}{2} n+\frac{35}{2} f-33 p+O(\log (n+f))$ if $p \leq \frac{n-f}{2}, R(n, p, f) \leq$ $18 n+34 f+O(\log (n+f))$, if $\frac{n-f}{2} \leq p \leq \frac{n+f}{2}$ and $R(n, p, f) \leq \frac{3}{2} n+\frac{35}{2} f+33 p+O(\log (n+f))$ if $p \geq \frac{n+f}{2}$. Finally, we give lower bounds for $R(n, 0, f)$ and $S^{+}(n)$ and show optimal networks for small value of $n$.
\end{abstract}

Key-words: network design, on-board network, fault tolerance, vulnerability

\footnotetext{
* The author was partially supported by the European project FET-CRESCCO
} 


\section{Repartiteurs, sélecteurs et supersélecteurs}

Résumé : Un $(n, p, n+f)$-réseau $G$ est un graphe $(V, E)$ dont l'ensemble de sommets $V$ est partitionné en quatre sous-ensembles $\mathcal{P}, \mathcal{I}, \mathcal{O}$ et $\mathcal{S}$ appelés respectivement les prioritiés, les entrées ordinaires, les sorties et les commutateurs, satisfaisant les contraintes suivantes : il y a $p$ priorités, $n-p$ entrées ordinaires et $n+f$ sorties; chaque priorité, entrée ordinaire ou sortie est reliée à exactement un commutateur; les commutateurs ont degré au plus 4 . Un $(n, p, n+f)$-réseau est un $(n, p, f)$-répartiteur si pour toute paire de sous-ensembles disjoints $\mathcal{F}$ et $\mathcal{B}$ de $\mathcal{O}$ avec $|\mathcal{F}|=f$ et $|\mathcal{B}|=p$, il existe dans $G, n$ chemins arêtes-disjoints, $p$ d'entre eux allant de $\mathcal{P}$ à $\mathcal{B}$ et les $n-p$ autres de $\mathcal{I}$ à $\mathcal{O} \backslash(\mathcal{B} \cup \mathcal{F})$. Le problème consiste à déterminer le nombre minimum $R(n, p, f)$ de commutateurs d'un $(n, p, f)$-répartiteur et de construire un répartiteur avec le moins possible de commutateurs.

Dans ce rapport, nous montrons comment construire des répartiteurs à partir de répartiteurs particuliers, nommément les $(n, 0, f)$-répartiteurs aussi appelés $(n, n+f)$-selecteurs. Nous construisons ensuite des sélecteurs par l'intermédiaire de réseaux plus puissants appelés supersélecteurs. Un $(n, 0, n)$-réseau est un $n$-supersélecteur si pour toute paire d'ensembles $\mathcal{I}^{\prime} \subset \mathcal{I}$ et $\mathcal{O}^{\prime} \subset \mathcal{O}$ vérifiant $\left|\mathcal{O}^{\prime}\right|=\left|\mathcal{I}^{\prime}\right|$, il existe dans $G,\left|\mathcal{O}^{\prime}\right|$ chemins arête-disjoints reliant $\mathcal{I}^{\prime}$ à $\mathcal{O}^{\prime}$. Nous montrons que le nombre minimum de commutateurs d'un $n$-supersélecteur, $S^{+}(n)$, est au plus $17 n+O(\log (n))$. Nous en déduisons ensuite que $R(n, p, f) \leq \frac{69}{2} n+\frac{35}{2} f-$ $33 p+O(\log (n+f))$ si $p \leq \frac{n-f}{2}, R(n, p, f) \leq 18 n+34 f+O(\log (n+f))$, si $\frac{n-f}{2} \leq p \leq \frac{n+f}{2}$ et $R(n, p, f) \leq \frac{3}{2} n+\frac{35}{2} f+33 p+O(\log (n+f))$ si $p \geq \frac{n+f}{2}$. Enfin, nous donnons des bornes inférieures pour $R(n, 0, f)$ et $S^{+}(n)$ et exhibons des réseaux optimaux pour de petites valeurs de $n$.

Mots-clés : conception de réseaux, réseau embarqué, tolérance aux pannes, vulnérabilité 


\section{Introduction}

Modern telecommunications satellites are very complex to design and an important industrial issue is to provide robustness at the lowest possible cost. A key component of telecommunication satellites is an interconnection network which allows to redirect signals received by the satellite to a set of amplifiers where the signals will be retransmitted. In this paper, we consider a certain type of interconnection network as asked by Alcatel Space Industries. The network is made of expensive switches ; so we want to minimize their number subject to the following conditions : Each input and output is adjacent to exactly one link ; each switch is adjacent to exactly four links ; there are $n$ inputs (signals) and $n+f$ outputs (amplifiers) ; among the $n+f$ outputs, $f$ can fail permanently; among the $n$ input signals, $p$ of them called priorities must be connected to the amplifiers providing the best quality of service (that is to some specific outputs) and the other signals should be sent to other amplifiers. Note that the priority signals are given, but the amplifiers providing the quality of service change during the life of the satellite and so the networks should be able to route the signals for any set of $f$ failed outputs and any set of $p$ best quality outputs.

This problem can be formally restated as follows:

Definition 1 An $(n, p, n+f)$-network $G$ is a graph $(V, E)$ where the vertex set $V$ is partitioned into four subsets $\mathcal{P}, \mathcal{I}, \mathcal{O}$ and $\mathcal{S}$ called respectively the priorities, the ordinary inputs, the outputs and the switches, satisfying the following constraints:

- there are $p$ priorities, $n-p$ ordinary inputs and $n+f$ outputs;

- each priority, each ordinary input and each output is connected to exactly one switch; - switches have degree at most 4.

An $(n, p, n+f)$-network is a $(n, p, f)$-repartitor if for any disjoint subsets $\mathcal{F}$ and $\mathcal{B}$ of $\mathcal{O}$ with $|\mathcal{F}|=f$ and $|\mathcal{B}|=p$, there exist in $G, n$ edge-disjoint paths, $p$ of them from $\mathcal{P}$ to $\mathcal{B}$ and the $n-p$ others joining $\mathcal{I}$ to $\mathcal{O} \backslash(\mathcal{B} \cup \mathcal{F})$. The set $\mathcal{F}$ corresponds to set of failures and $\mathcal{B}$ to the set of amplifiers providing the best quality of service. We denote $R(n, p, f)$ the minimum number of switches (i.e. cardinality of $\mathcal{S}$ ) of a valid $(n, p, f)$-repartitor. A $(n, p, f)$-repartitor with $R(n, p, f)$ switches will be called minimum.

Problem 1 Determine $R(n, p, f)$ and construct a minimum (or almost minimum) repartitor.

A particular case of repartitors are those with only one type of inputs, i.e. $(n, 0, f)$ repartitors (or $(n, n, f)$-repartitors) also called $(n, n+f)$-selectors. A $(p, n)$-selector is a network with a set $\mathcal{I}$ of $p$ inputs and a set $\mathcal{O}$ of $n$ outputs, such that for any subset $\mathcal{O}^{\prime}$ of $p$ outputs there exists a set of $p$ edge-disjoint paths connecting $\mathcal{I}$ to $\mathcal{O}^{\prime}$.

In [4] and [6], $(n, n+f)$-selectors) with $f$ fixed, were studied. Let us denote $S(p, n)$ the minimum number of switches (i.e. cardinality of $\mathcal{S}$ ) of a $(p, n)$-selector. In [4], it is shown that $S(n, n+2)=R(n, 0,2)=n$. In [6], the following values for small $f$ are also given : $S(n, n+4)=R(n, 0,4)=n+\left\lceil\frac{n}{4}\right\rceil ; S(n, n+6)=R(n, 0,6)=n+\frac{n}{4}+\sqrt{\frac{n}{8}}+O(1) ; S(n, n+8)=$ $R(n, 0,8)=n+\frac{n}{3}+\frac{2}{3} \sqrt{\frac{n}{3}}+O(\sqrt[4]{n})$ and $S(n, n+12)=R(n, 0,12)=n+\frac{3 n}{7}+O(\sqrt{n})$. 
It is also proved that $\frac{3 n}{2}-O\left(\frac{n}{f}\right) \leq S(n, n+f)=R(n, 0, f) \leq \frac{3 n}{2}+g(f)$ with $g$ a function depending only on $f$. However, this function is exponential in $f$, hence if $\log (n)=o(f)$ this upper bound is very bad.

In [5], it is shown that $R(n, p, 0) \leq n-p+\frac{n}{2}\left\lceil\log _{2} p\right\rceil$. Moreover, some exact values of $R(n, p, f)$ were given when $p$ and $f$ are small.

The main objective of this paper (sectionconstruction) is to prove an upper bound for $R(n, p, f)$ which is linear in $n, p$ and $f$.

We first show Subsection 2.1 how to construct a repartitor from two selectors and derive the inequality $R(n, p, f) \leq S(p, n+f)+S(n-p, n+f)+n$. We then focus on selectors.

Selectors are an anloguous of (the opposite of) concentrators (see [7]). An (n,m)concentrator is a directed acyclic graph with $n$ distinguished vertices called inputs and a disjoint set of $m$ distinguished vertices outputs such that for any subset $A$ of $m$ inputs there exists a set of $m$ vertex-disjoint paths connecting $A$ to the outputs. Lots of papers are devoted to study the minimum number of edges of an $(n, m)$-concentrator.

Here we want to minimize the number of switches of selectors which is very close to minimize the number of edges since every switch has degree at most 4 , so the number of edges of a minimum $(p, n)$-selector is at most $\frac{1}{2}(4 S(p, n)+n+p)$.

In order to build selectors, we first build more powerful networks called superselectors. An $(n, 0, n)$-network is an $n$-superselector if for any subsets $\mathcal{I}^{\prime} \subset \mathcal{I}$ and $\mathcal{O}^{\prime} \subset \mathcal{O}$ with $\left|\mathcal{O}^{\prime}\right|=\left|\mathcal{I}^{\prime}\right|$, there exist in $G,\left|\mathcal{O}^{\prime}\right|$ edge-disjoint paths joining $\mathcal{I}^{\prime}$ to $\mathcal{O}^{\prime}$. We will denote $S^{+}(n)$ the minimum number of switches (i.e. cardinality of $\mathcal{S}$ ) of a $n$-superselector. A $n$-superselector with $S^{+}(n)$ switches will be called minimum.

Superselectors are very powerful since they are like $(p, n)$-selectors for every $1 \leq p \leq n$. Indeed, it is easy to see that the network obtained from a $n$-superselector by deleting any set of $n-p$ inputs is a $(p, n)$-selector.

Proposition 1 For any $p \leq n, S(p, n) \leq S^{+}(n)$.

Then we give two constructions of superselectors. The first one (Subsection 2.2) uses a technique inspired by Pippenger's construction [8] of superconcentrators. It yields an $n$ superselector with $17 n+O(\log (n))$ switches. However, this construction is based on the existence of funnels which is proved in Lemma 2 in a non constructive way. Hence, we give Subsection 2.3 an explicit construction of an $n$-superselectors with $20 n+O(1)$ switches. It is based an a result of Alon and Capalbo [1]. Then, in Subsection 2.4, we give constructions of selectors from superselectors. We show :

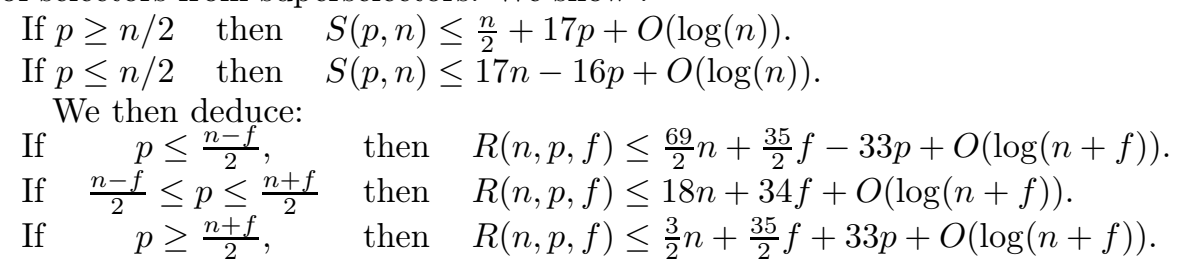

In the second part of this paper, we study lower bounds to the number of switches in a selector or superselector. In Subsection 3.1, we prove that: 
if $p$ is even then $S(p, n) \geq \frac{2^{p / 2}-1}{2^{p / 2}} n+\Theta(1)$;

if $p$ is odd then $S(p, n) \geq \frac{2^{(p+1) / 2}-3}{2^{(p+1) / 2}} n+\Theta(1)$.

We conjecture that equality holds. We establish it for $p \leq 6$ in Subsection 3.2. In Subsection 3.3, we give lower bounds for $S^{+}(n)$ and in Subsection 3.4, we show optimal superselectors for small values of $n$.

\section{Construction of superselectors, selectors and reparti- tors}

\subsection{Constructing repartitors with selectors}

Lemma $1 R(n, p, f) \leq S(p, n+f)+S(n-p, n+f)+n+f$

Proof. Let $S$ be a $(p, n+f)$-selector with output-set $\left\{o_{1}, o_{2}, \ldots, o_{n+f}\right\}$ and $S^{\prime}$ an $(n-$ $p, n+f)$-selector with output-set $\left\{o_{1}^{\prime}, o_{2}^{\prime}, \ldots, o_{n+f}^{\prime}\right\}$. Let $H$ be the $(n, p, n+f)$-network constructed from $S$ and $S^{\prime}$ by replacing each pair $\left\{o_{i}, o_{i}^{\prime}\right\}, 1 \leq i \leq n+f$, by a switch $s_{i}$ adjacent to an output $q_{i}$ and the neighbours of $o_{i}$ and $o_{i}^{\prime}$. See Figure 1. The priorities of $H$

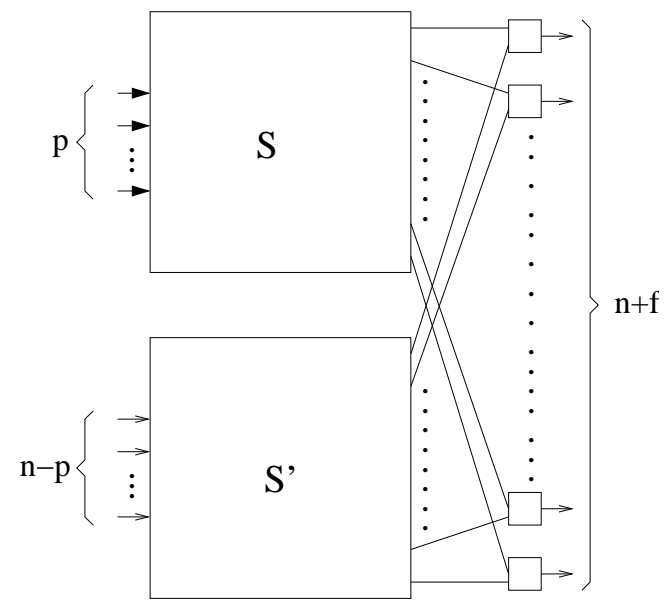

Figure 1: Construction of an $(n, p, f)$-repartitor from a $(p, n+f)$ - and an $(n-p, n+f)$-selector

are the inputs of $S$ and its ordinary inputs the inputs of $S^{\prime}$. It is easy to check that $H$ is a $(n, p, 0)$-repartitor. Indeed the priorities are routed through $S$, the ordinary inputs through $S^{\prime}$ and the switches $s_{i}$ allow us to select a priority path or an ordinary one.

$\mathrm{RR} \mathrm{n}^{\circ} 5686$ 
From an $(n, p, f)$-repartitor, one can easily construct a $(p, n+f)$-selector or an $(n-$ $p, n+f)$-selector by removing inputs and outputs. So $S(p, n+f)$ and $S(n-p, n+f)$ are less or equal to $R(n, p, f)$. Hence, if a $(n, p, f)$-repartitor is constructed from two optimum selectors using the above construction, it has at most $2 R(n, p, f)+n+f$ switches. So finding minimum (or almost minimum) selectors will give us fairly small repartitors. Hence in the remaining of the paper, we will focus on selectors.

In order to construct selectors, we first construct more powerful networks: the superselectors.

\subsection{Non explicit construction of superselectors}

We first give a recursive construction for superselectors. Therefore we need a preliminary lemma due to Pippenger [8].

Let $\theta$ be the function from $\mathbb{N}$ into $\mathbb{N}$ defined by $\theta(n)=4\left\lceil\frac{n}{6}\right\rceil$.

Lemma 2 (Pippenger [8]) For every $n$, there is a bipartite graph Bip $p_{n}=(A, B)$ with $|A|=n$ and $|B|=\theta(n)$, in which every vertex of $A$ has outdegree 6 , every vertex of $B$ has indegree 9, and, for every $k \leq n / 2$ and every set $S \subset$ of $k$ vertices, there exists a matching saturating $S$.

\section{Theorem 1}

$$
S^{+}(n) \leq 17 n+O(\log n)
$$

Proof. Let $S_{n}$ be the $(n, 0, n)$-network defined recursively as follows : If $n \leq 4$, let $S_{n}$ be the (optimum) superselector defined Subsection 3.4.

If $n \geq 6$, let $B_{i p_{n}}$ be the bipartite graph defined in Lemma 2 with $A=\left\{a_{1}, \ldots, a_{n}\right\}$ and $B=\left\{b_{1}, \ldots, b_{\theta(n)}\right\}$ and $\left\{i_{j}^{\prime}, 1 \leq j \leq \theta(n)\right\}$ and $\left\{o_{j}^{\prime}, 1 \leq j \leq \theta(n)\right\}$ be the input and output sets of $S_{\theta(n)}$. Let us construct $S_{n}$ as follows (See Figure 2) : For $1 \leq l \leq n$, replace the vertex $a_{l}$ by an path $A_{l}=\left(u_{l}^{1}, u_{l}^{2}, u_{l}^{3}\right)$ incident to the 6 edges of $B i p_{n}$ incident to $a_{l}$ (1 incident to $u_{l}^{1}, 2$ incident to $u_{l}^{2}$ and 3 incident to $u_{l}^{3}$ ) and connect $u_{l}^{1}$ to an input $i_{l}$ and an output $o_{l}$. For $1 \leq l \leq \theta(n)$, replace the vertex $b_{l}$ by an path $B_{l}=\left(v_{l}^{1}, v_{l}^{2}, v_{l}^{3}, v_{l}^{4}\right)$ incident to the 9 edges of $B i p_{n}$ incident to $b_{l}$ (3 incident to $v_{l}^{1}$ and 2 to each other switch), identify $v_{l}^{4}$ with $i_{l}^{\prime}$ and $o_{l}^{\prime}$. This is possible since $i_{l}^{\prime}$ and $o_{l}^{\prime}$ are adjacent to the same switch (corresponding to $u_{l}^{1}$ ) by construction.

Let us prove by induction on $n$ that $S_{n}$ is an $n$-superselector.

Let $\mathcal{I}^{\prime}$ and $\mathcal{O}^{\prime}$ be sets of inputs and outputs with the same cardinality. Let $I_{i}=\left\{j, i_{j} \in \mathcal{I}^{\prime}\right\}$ and $I_{o}=\left\{j, o_{j} \in \mathcal{I}^{\prime}\right\}$. For $j \in I_{i} \cap I_{o}$, let $P_{j}=\left(i_{j}, a_{j}^{1}, o_{j}\right)$. Let $J_{i}=I_{i} \backslash I_{o}$ and $J_{o}=I_{o} \backslash I_{i}$. It remains to find paths from the inputs $i_{j}, j \in J_{i}$ to outputs $o_{j}, j \in J_{o}$.

By Lemma 2, in Bip $p_{n}$, there is a matching $M_{i}$ which saturates $\left\{a_{j}, j \in J_{i}\right\}$ and a matching $M_{o}$ which saturates $\left\{a_{j}, j \in J_{o}\right\}$. Note that since $J_{i}$ and $J_{o}$ are disjoint $M_{i} \cap M_{o}=\emptyset$. For $j \in J_{i}$, let $g(j)$ be the integer such that $a_{j} b_{g(j)} \in M_{i}$ and for $j \in J_{o}$, let $h(j)$ be the integer such that $a_{j} b_{h(j)} \in M_{o}$. 


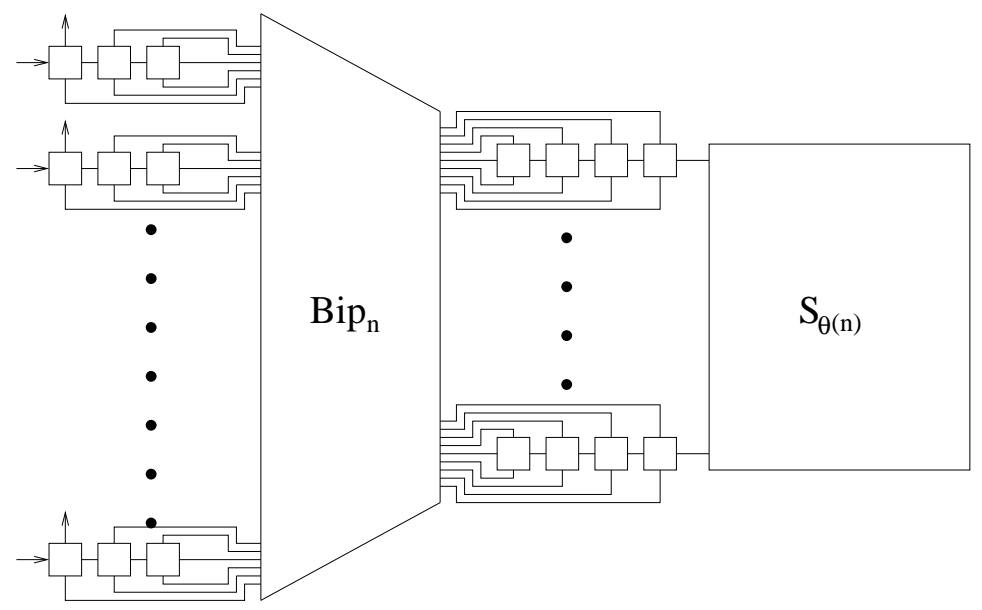

Figure 2: The network $S_{n}$

Set $L=g\left(J_{i}\right) \cap h\left(J_{o}\right), L_{i}=g\left(J_{i}\right) \backslash L$ and $L_{o}=h\left(J_{o}\right) \backslash L$. Since $S_{\theta(n)}$ is a $\theta(n)$ superselector. There is a set $\mathcal{R}$ of edge-disjoint paths joining $\left\{v_{l}^{4}, l \in L_{i}\right\}$ to $\left\{v_{l}^{4}, l \in L_{o}\right\}$. For every $l \in L_{i}$, let $R_{l}$ be the path of $\mathcal{R}$ joining $v_{l}^{4}$ to a vertex $v_{f(l)}^{4}$ with $f(l) \in L_{o}$.

Let $j \in J_{i}$.

If $g(j) \notin L$, let $j^{\prime}=h^{-1}[f(g(j))]$. There is a path $Q_{j}, j \in J_{i}$ corresponding to $a_{j} b_{g(j)}$ from $i_{j}$ to a vertex $v_{g(j)}^{4}$ and a path $Q_{j}^{\prime}$ from $v_{h\left(j^{\prime}\right)}^{4}$ to $o_{j^{\prime}}$ corresponding to the edge $a_{j^{\prime}} b_{h\left(j^{\prime}\right)}$. Hence $P_{j}=Q_{J} R_{j} Q_{j}^{\prime}$ route $i_{j}$ to $o_{j^{\prime}}$.

If $g(j) \in L$, let $j^{\prime} \in J_{o}$ such that $h\left(j^{\prime}\right)=g(j)$. By construction of $S_{n}$, there is a path $Q_{j}, j \in J_{i}$ corresponding to $a_{j} b_{g(j)}$ from $i_{j}$ to a vertex $x$ of $B_{g(j)}$ and a path $Q_{j}^{\prime}$ from $x$ to $o_{j^{\prime}}$ corresponding to the edge $a_{j^{\prime}} b_{h\left(j^{\prime}\right)}$. Hence $P_{j}=Q_{J} Q_{j}^{\prime}$ route $i_{j}$ to $o_{j^{\prime}}$.

Obviously the $P_{j}, j \in I_{i}$, are edge disjoint since a path $A_{j}$ or $B_{l}$ intersects at most one of them. So they are the desired paths.

Now $\left|S_{n}\right| \leq 3 n+4 \theta(n)+\left|S_{\theta(n)}\right|$. For $n \geq 4$, define $\theta^{0}(n)=n$ and $\theta^{t+1}(n)=\theta\left(\theta^{t}(n)\right)$. Pick $t$ the smallest integer such that $\theta^{t+1}(n)=4$. Since $\frac{2}{3}<1$, then $t=O(\log n)$ since $\frac{2}{3}<1$. Now applying the above equation $t+1$ times, we get

$$
\left|S_{n}\right| \leq 3 n+7\left(\theta^{1}(n)+\cdots+\theta^{t}(n)\right)+16+S^{+}(4)
$$

It is easy to show by induction on $t$ that $\theta^{t}(n) \leq\left(\frac{2}{3}\right)^{t} n+8$. This implies that

$$
\left|S_{n}\right| \leq 17 n+56(t+1)+O(1) \leq 17 n+O(\log (n))
$$




\subsection{Explicit construction for superselectors}

In order to construct explicitely an $n$-superselector, we need the following Lemma due to Alon and Capalbo [1]:

Lemma 3 (Alon and Capalbo [1]) There exists an explicit 10-regular bipartite graph $H_{n}=$ $(A, B)$ with $A=\left\{a_{1}, a_{2}, \ldots, a_{n}\right\}, B=\left\{b_{1}, b_{2}, \ldots, b_{n}\right\}$ and $n=k \times 2^{l+1}$ with $k=63 \times 64 \times 65$ such that for any subsets $A_{1}$ and $A_{2}$ of $A$ of the same cardinality $\left|A_{1}\right|=\left|A_{2}\right|$, there exists two matchings $M_{1}$ and $M_{2}$ such that:

(i) $M_{1}$ saturates $A_{1}$ and $M_{2}$ saturates $A_{2}$;

(ii) Let $i$ be any integer in $\{1,2, \ldots, n / 2\}$. Then if $M_{1}$ covers $b_{i}$ and $b_{i+n / 2}$ then $M_{2}$ covers at least one vertex of $\left\{b_{i}, b_{i+n / 2}\right\}$. Similarly, if $M_{2}$ covers $b_{i}$ and $b_{i+n / 2}$ then $M_{1}$ covers at least one vertex of $\left\{b_{i}, b_{i+n / 2}\right\}$.

Using this bipartite graph and a method similar to Alon and Capalbo's one for superconcentrators, we construct explicitely superselector recursively :

Theorem 2 There exists an explicit $n$-superselector $20 n+O(1)$ switches.

Proof. Let $N_{n}$ be the network constructed recursively as follows. Take the bipartite graph $H_{n}$ constructed in Lemma 3 and $N_{n / 2}$ with input set $\left\{i_{1}^{\prime}, i_{2}^{\prime}, \ldots, i_{n / 2}^{\prime}\right\}$ and output set and $\left\{o_{1}^{\prime}, o_{2}^{\prime}, \ldots, o_{n / 2}^{\prime}\right\}$.

For $1 \leq j \leq n$, replace the vertices $a_{j}$ by a path $A_{j}=\left(u_{j}^{1}, u_{j}^{2}, u_{j}^{3}, u_{j}^{4}, u_{j}^{5}\right)$ incident to the 10 edges of $H_{n}$ incident to $a_{j}$ (one incident to $u_{j}^{1}$, two incident to each of $u_{j}^{2}, u_{j}^{3}$, $u_{j}^{4}$ and three incident to $u_{j}^{5}$ ) and connect $u_{j}^{1}$ to an input $i_{j}$ and an output $o_{j}$.

For $1 \leq l \leq n / 2$, replace the two vertices $b_{l}$ and $b_{l+n / 2}$ by a path $B_{l}=\left(v_{l}^{1}, v_{l}^{2}, \ldots, v_{l}^{10}\right)$ incident to the 20 edges of $H_{n}$ incident to $b_{l}$ or $b_{l+n / 2}$, (Each switch is incident to one edge of incident to $b_{l}$ and one edge incident to $\left.b_{l+n / 2}\right)$. Finally, for $1 \leq l \leq n / 2$, remove $i_{l}^{\prime}$ and $o_{l}^{\prime}$ and connect their common neighbour $s_{l}^{\prime}$ in $N^{\prime}$ with $v_{l}^{1}$ and $v_{l}^{10}$. The network $N_{n}$ is depicted Figure 3.

Let us prove by induction that $N_{n}$ is an $n$-superselector.

Let $\mathcal{I}^{\prime} \subset \mathcal{I}$ and $\mathcal{O}^{\prime} \subset \mathcal{O}$ be two sets of the same cardinality $k$. Set $I_{1}=\left\{j, i_{j} \in \mathcal{I}^{\prime}\right\}$, $I_{2}=\left\{j, i_{j} \in \mathcal{I}^{\prime}\right\}, I=I_{1} \cap I_{2}, J_{1}=I_{1} \backslash I$ and $J_{2}=I_{2} \backslash I$. If $j \in I$, route $i_{j}$ to $o_{j}$ via $P_{j}=\left(i_{j}, v_{j}^{1}, o^{j}\right)$. It remains to find paths from $\left\{i_{j}, j \in J_{1}\right\}$ to $\left\{o_{j}, j \in J_{2}\right\}$.

Let $A_{1}=\left\{a_{j}, j \in J_{1}\right\}$ and $A_{2}=\left\{a_{j}, j \in J_{2}\right\}$, and $M_{1}$ and $M_{2}$ be the two matchings constructed as in Lemma 3. For $j \in J_{i}$, let $g(j)$ be the integer such that an edge $e$ among $a_{j} b_{g(j)}$ and $a_{j} b_{g(j)+n / 2}$ is in $M_{1}$ and let $u_{j}^{\prime} v_{j}^{\prime}$ the edge of $N_{n}$ corresponding to the edge $e$. By construction $u_{j}^{\prime} \in V\left(A_{j}\right)$ and $v_{j}^{\prime} \in V\left(B_{g(j)}\right)$. Let $Q_{j}^{\prime}$ be the path from $i_{j}$ to $v_{j}^{\prime}$ in $A_{j} \cup e$. For $j \in J_{o}$, let $h(j)$ be the integer such that an edge $e$ among $a_{j} b_{h(j)}$ and $a_{j} b_{h(j)+n / 2}$ is in $M_{2}$ and let $u_{j}^{\prime \prime} v_{j}^{\prime \prime}$ the edge of $N_{n}$ corresponding to the edge $e$ and $Q_{j}^{\prime \prime}$ the path from $v_{j}^{\prime \prime}$ to $o_{j}$. For $0 \leq a, b \leq 2, L_{a, b}$ be set set of integers $l$ such that $\left|g^{-1}(l)\right|=a$ and $\left|h^{-1}(l)\right|=b$. By the property (ii) of $M_{1}$ and $M_{2}, L_{2,0}$ and $L_{0,2}$ are empty. 


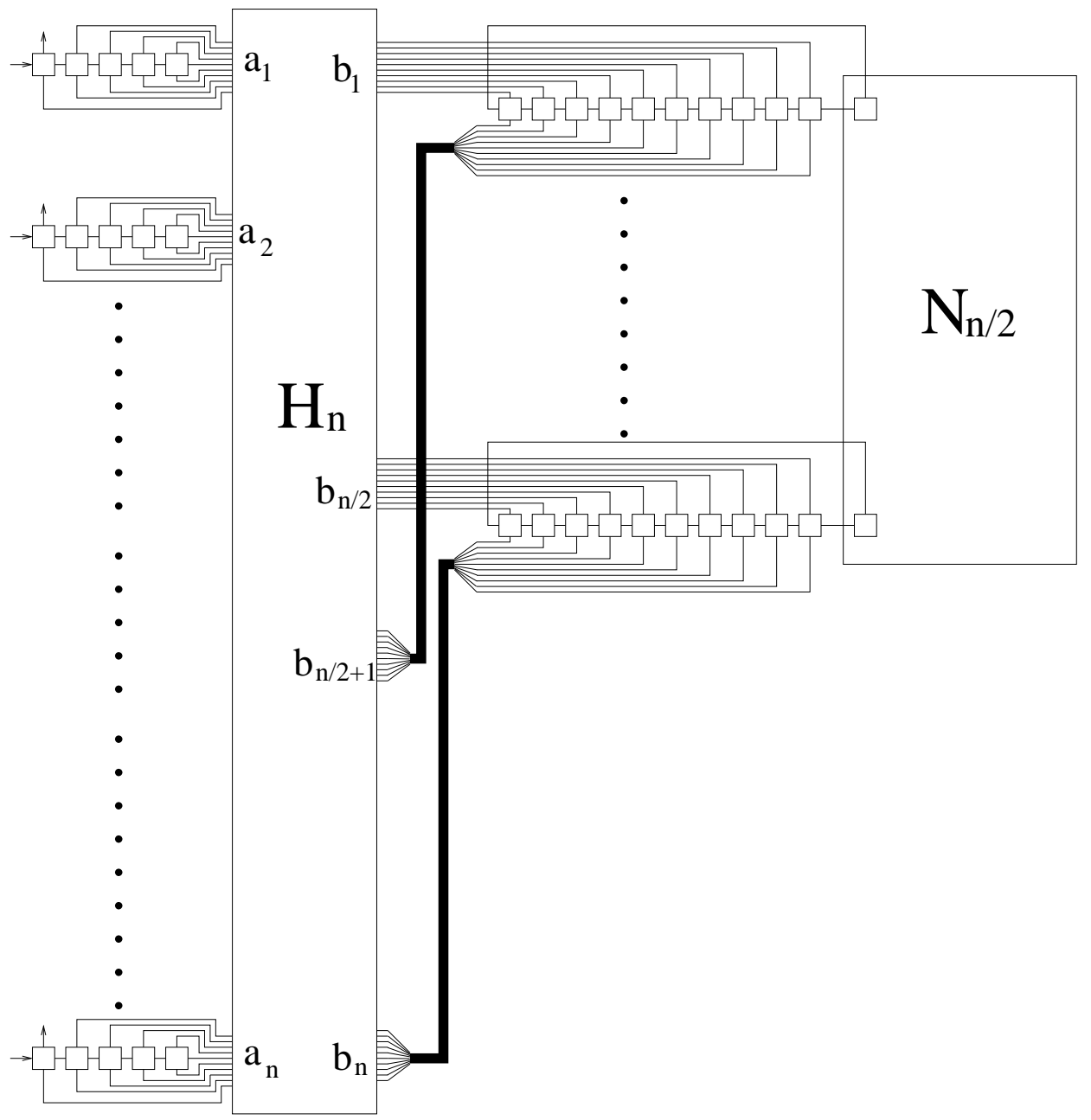

Figure 3: The network $N_{n}$. 
If $l \in L_{2,2}$, set $\left\{j, j^{\prime}\right\}=g^{-1}(l)$ and $\left\{k, k^{\prime}\right\}=h^{-1}(l)$. In the cycle induced by $V\left(B_{l}\right) \cup s_{l}^{\prime}$ there exist two edge-disjoint paths joining $\left\{v_{j}^{\prime}, v_{j^{\prime}}^{\prime}\right\}$ to $\left\{v_{k}^{\prime \prime}, v_{k^{\prime}}^{\prime \prime}\right\}$. Free to exchange $j$ and $j^{\prime}$ we may assume that a path $Q_{j}$ goes from $v_{j}^{\prime}$ to $v_{k}^{\prime \prime}$ and another $Q_{j^{\prime}}$ goes from $v_{j^{\prime}}^{\prime}$ to $v_{k^{\prime}}^{\prime \prime}$. Then route $i_{j}$ to $o_{k}$ via $P_{j}=Q_{j}^{\prime} Q_{j} Q_{k}^{\prime \prime}$ and $i_{j^{\prime}}$ to $o_{k^{\prime}}$ via $P_{j^{\prime}}=Q_{j^{\prime}}^{\prime} Q_{j^{\prime}} Q_{k^{\prime}}^{\prime \prime}$.

If $l \in L_{1,1}$, set $\{j\}=g^{-1}(l)$ and $\{k\}=h^{-1}(l)$. There is a subpath $Q_{j}$ of $B_{l}$ which goes from $v_{j}^{\prime}$ to $v_{k}^{\prime \prime}$. Then route $i_{j}$ to $o_{k}$ via $P_{j}=Q_{j}^{\prime} Q_{j} Q_{k}^{\prime \prime}$.

If $l \in L_{2,1}$, set $\left\{j, j^{\prime}\right\}=g^{-1}(l)$ and $\{k\}=h^{-1}(l)$. In the cycle induced by $V\left(B_{l}\right) \cup s_{l}^{\prime}$ there exist two edge-disjoint paths $Q_{j_{l}}$ from $v_{j_{l}}^{\prime}$ to $s_{l}^{\prime}$ and the other $Q_{j_{l}^{\prime}}$ from $v_{j_{l}}^{\prime}$ to $v_{k}^{\prime \prime}$. Route $i_{j_{l}^{\prime}}$ to $o_{k}$ via $P_{j_{l}^{\prime}}=Q_{j_{l}^{\prime}}^{\prime} Q_{j_{l}^{\prime}} Q_{k}^{\prime \prime}$.

If $l \in L_{1,0}$, set $\{j\}=g^{-1}(l)$. There is a subpath $Q_{j}$ of $B_{l}$ which goes from $v_{j}^{\prime}$ to $s_{l}^{\prime}$.

If $l \in L_{1,2}$, set $\{j\}=g^{-1}(l)$ and $\left\{k, k^{\prime}\right\}=h^{-1}(l)$. In the cycle induced by $V\left(B_{l}\right) \cup s_{l}^{\prime}$ there exist two edge-disjoint paths $Q_{k_{l}}$ from $s_{l}^{\prime}$ to $v_{k_{l}}^{\prime \prime}$ and the other $Q_{k_{l}^{\prime}}$ from $v_{j}^{\prime}$ to $v_{k_{l}^{\prime}}^{\prime \prime}$. Route $i_{j}$ to $o_{k_{l}^{\prime}}$ via $P_{j}=Q_{j}^{\prime} Q_{k_{l}} Q_{k_{l}^{\prime}}^{\prime \prime}$.

If $l \in L_{0,1}$, set $\{k\}=h^{-1}(l)$. There is a subpath $Q_{k}$ of $B_{l}$ which goes from $s_{l}^{\prime}$ to $v_{k}^{\prime \prime}$.

Now, since $N_{n / 2}$ is an $(n / 2)$-superselector, in $N_{n / 2}$, there is a set $\mathcal{R}$ edge-disjoint paths joining $\left\{s_{l}^{\prime}, l \in L_{2,1} \cup L_{1,0}\right\}$ to $\left\{s_{l}^{\prime}, l \in L_{1,2} \cup L_{0,1}\right\}$. For $l \in L_{2,1} \cup L_{1,0}$, let $R_{l}$ be the path of $\mathcal{R}$ with end $s_{l}^{\prime}$ and let $s_{f(l)}^{\prime}$ be its other end.

For $j \in\left\{j_{l}, l \in L_{2,1}\right\} \cup g^{-1}\left(L_{1,0}\right)$, let $l=f(g(j))$, and $k=k_{l}$ if $l \in L_{1,2}$ and $k=h^{-1}(l)$ otherwise. Then route $i_{j}$ to $o_{k}$ via $P_{j}=Q_{j}^{\prime} Q_{j} R_{g(j)} Q_{k}^{\prime \prime}$.

Oviously $N_{n}$ has $\left|N_{n / 2}\right|+10 n$ switches. So by induction, one can explicitly construct an $n$-superselector with at most $20 n+O(1)$ switches.

\subsection{Applications to selectors and repartitors}

Theorem 1 and Proposition 1 yield $S(p, n) \leq 17 n+O(\log (n))$. However, better upper bounds may be achieved by constructing a $(p, n)$-selector from smaller superselectors.

\section{Theorem 3}

$$
\begin{aligned}
& \text { If } p \geq n / 2 \quad \text { then } \quad S(p, n) \leq \frac{n}{2}+17 p+O(\log (n)) . \\
& \text { If } p \leq n / 2 \quad \text { then } \quad S(p, n) \leq 17 n-16 p+O(\log (n)) .
\end{aligned}
$$

Proof. Suppose first that $p \geq n / 2$. Let $G(n, p)$ be the $(p, 0, n)$-network constructed as follows from $S_{p}$ be the $p$-superselector constructed in Subsection 2.2 with input set $\left\{i_{1}^{\prime}, \ldots, i_{p}^{\prime}\right\}$ and output set $\left\{o_{1}^{\prime}, \ldots, o_{p}^{\prime}\right\}$. For $1 \leq j \leq\lceil n / 2\rceil$, create a switch $t_{j}$ connected to an input $i_{j}$ and two outputs $o_{j}$ and $o_{n+1-j}$ (except eventually $t_{\lceil n / 2\rceil}$ which is adjacent to a unique output $o_{j}$ if $n$ is odd), remove $i_{j}^{\prime}$ and $o_{j}^{\prime}$ and connect $t_{j}$ to their common neighbour $v_{j}$. For $\lceil n / 2\rceil+1 \leq j \leq p$, let $i_{j}^{\prime}$ be the input $i_{j}$ of $G$ and remove $o_{j}^{\prime}$. See Figure 4 left.

Let us prove that $G(n, p)$ is a $(p, n)$-selector. Let $\mathcal{I}^{\prime}$ and $\mathcal{O}^{\prime}$ be set of inputs and outputs of $G(n, p)$ with the same cardinality. Set $I=\left\{j,\left\{o_{j}, o_{n+1-j}\right\} \subset \mathcal{O}^{\prime}\right\}, I_{1}=\left\{j,\left\{o_{j}, o_{n+1-j}\right\} \cap\right.$ $\left.\mathcal{O}^{\prime}=\left\{o_{j}\right\}\right\}, I_{2}=\left\{j,\left\{o_{j}, o_{n+1-j}\right\} \cap \mathcal{O}^{\prime}=\left\{o_{n+1-j}\right\}\right\}$, and $J=\left\{j,\left\{o_{j}, o_{n+1-j}\right\} \cap \mathcal{O}^{\prime}=\right.$ $\emptyset\} \cup\{\lceil n / 2\rceil+1, \ldots, p\}$. If $j \in I_{1}$ then route $i_{j}$ to $o_{j}$ via $t_{j}$, and $j \in I_{2} \cup I$ then route $i_{j}$ to 

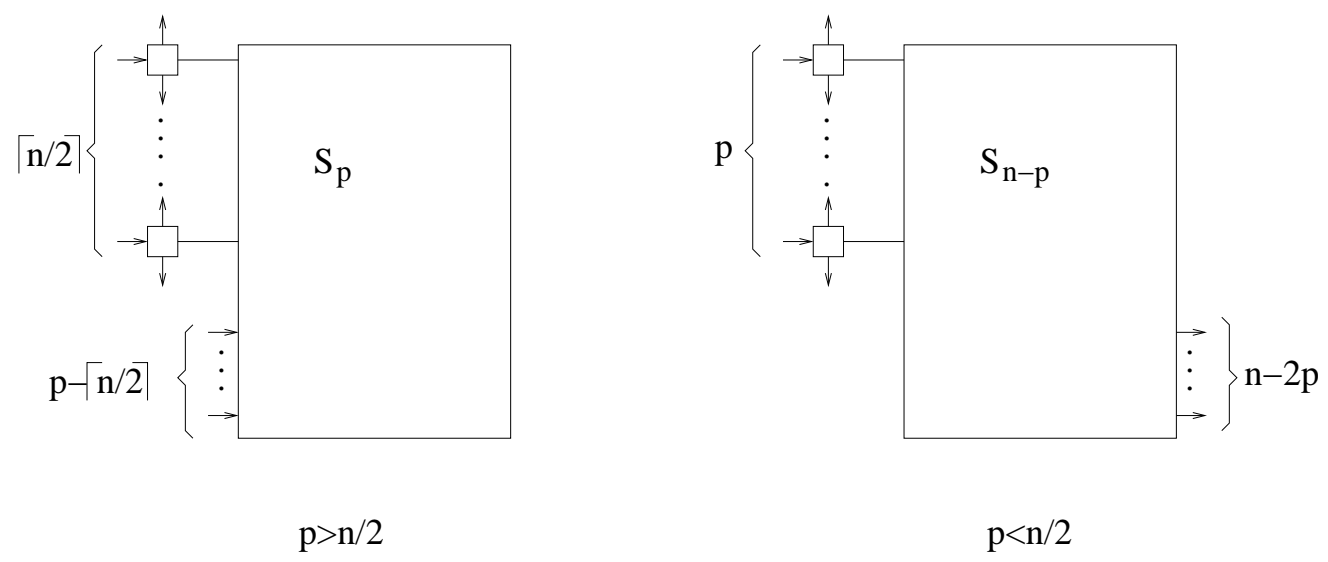

Figure 4: Construction of a $(p, n)$-selector from $S_{p}$ or $S_{n-p}$

$o_{n+1-j}$ via $t_{j}$. It remains to route the inputs $i_{j}, j \in J$ to outputs $o_{j}, j \in I$. This is possible through $S_{p}$ because it is a $p$-superselector and $t_{j}$ is adjacent to the common neighbour of $i_{j}^{\prime}$ and $o_{j}^{\prime}$ and then may be identified to each of these two.

Suppose now that $p \geq n / 2$. Let $G(n, p)$ be the $(p, 0, n)$-network constructed as follows from the $(n-p)$-superselector $S_{n-p}$ constructed in Subsection 2.2 with input set $\left\{i_{1}^{\prime}, \ldots, i_{p}^{\prime}\right\}$ and output set $\left\{o_{1}^{\prime}, \ldots, o_{p}^{\prime}\right\}$. For $1 \leq j \leq p$, create a switch $t_{j}$ connected to an input $i_{j}$ and two outputs $o_{j}$ and $o_{n+1-j}$, remove $i_{j}^{\prime}$ and $o_{j}^{\prime}$ and connect $t_{j}$ to their common neighbour $v_{j}$. For $2 p+1 \leq j \leq n-p$, remove $i_{j}^{\prime}$ and set $o_{j}=o_{j}^{\prime}$. See Figure 4 right.

Let us prove that $G(n, p)$ is a $(p, n)$-selector. Let $\mathcal{I}^{\prime}$ and $\mathcal{O}^{\prime}$ be set of inputs and outputs of $G(n, p)$ with the same cardinality. Set $I=\left\{j,\left\{o_{j}, o_{n+1-j}\right\} \subset \mathcal{O}^{\prime}\right\} \cup\{2 p+1, \ldots, n-p\}$, $I_{1}=\left\{j,\left\{o_{j}, o_{n+1-j}\right\} \cap \mathcal{O}^{\prime}=\left\{o_{j}\right\}\right\}, I_{2}=\left\{j,\left\{o_{j}, o_{n+1-j}\right\} \cap \mathcal{O}^{\prime}=\left\{o_{n+1-j}\right\}\right\}$, and $J=$ $\left\{j,\left\{o_{j}, o_{n+1-j}\right\} \cap \mathcal{O}^{\prime}=\emptyset\right\}$. If $j \in I_{1}$ then route $i_{j}$ to $o_{j}$ via $t_{j}$, and $j \in I_{2} \cup I$ then route $i_{j}$ to $o_{n+1-j}$ via $t_{j}$. It remains to route the inputs $i_{j}, j \in J$ to outputs $o_{j}, j \in I$. This is possible through $S_{n-p}$ because it is a $(n-p)$-superselector and $t_{j}$ is adjacent to the common neighbour of $i_{j}^{\prime}$ and $o_{j}^{\prime}$ and then may be identified to each of these two.

From Proposition 1 and Theorem 3, we derive the following upper bounds of $R(n, p, f)$.

\section{Corollary 1}

$$
\begin{array}{llll}
\text { If } & p \leq \frac{n-f}{2}, & \text { then } & R(n, p, f) \leq \frac{69}{2} n+\frac{35}{2} f-33 p+O(\log (n+f)) . \\
\text { If } & \frac{n-f}{2} \leq p \leq \frac{n+f}{2} & \text { then } & R(n, p, f) \leq 18 n+34 f+O(\log (n+f)) . \\
\text { If } & p \geq \frac{n+f}{2}, & \text { then } & R(n, p, f) \leq \frac{3}{2} n+\frac{35}{2} f+33 p+O(\log (n+f)) .
\end{array}
$$

Analogously, to Theorem 3, replacing $S_{n}$ by $N_{n}$ in the construction one can explicitly construct $(p, n)$-selectors with $20 n+19 p$ switches if $p \geq n / 2$ and $n / 2+20 p$ switches if 
$p \geq n / 2$. We then can derive $(n, p, f)$-repartitor with $20 n+20 f-19 p$ if $p \leq \frac{n-f}{2}, 21 n+40 f$ switches if $\frac{n-f}{2} \leq p \leq \frac{n+f}{2}$, and $n+20 f+19 p$ if $p \geq \frac{n+f}{2}$.

\section{Some lower bounds and minimum networks}

\subsection{Lower bounds for selectors}

Let $W$ be a set of vertices of a network. We denote by $i n(W)(\operatorname{resp}$. out $(W), s w(W))$ the number of inputs (resp. outputs, switches) in $W$. An edge connecting $W$ and $\bar{W}=V \backslash W$ is said to be $W$-cutting. The set of $W$-cutting edges is denoted by $\Delta(W)$ and its cardinality is denoted by $\operatorname{deg}(W)$.

Proposition $2 A(p, 0, n)$-network is a $(p, n)$-selector if and only if for every subset $W$ :

$$
\operatorname{deg}(W) \geq \min \{p, \operatorname{out}(W)\}-\operatorname{in}(W)
$$

Proof. Let $\mathcal{O}^{\prime}$ be a fixed set of $p$ outputs and let $o u t^{\prime}(W)=\left|W \cap \mathcal{O}^{\prime}\right|$. A variant of the Ford-Fulkerson Theorem states that the problem is feasible if and only if

$$
\forall W \subset V: \operatorname{deg}(W) \geq \operatorname{demand}(W)=\operatorname{out}^{\prime}(W)-i n(W) .
$$

It follows that a $(p, n)$-network is $(p, n)$-selector if and only if:

$$
\forall W \subset V: \operatorname{deg}(W) \geq \max \left\{\operatorname{out}^{\prime}(W) \mid \mathcal{O}^{\prime} \text { set of } p \text { outputs }\right\}-i n(W) .
$$

Now $\max \left\{\operatorname{out}^{\prime}(W) \mid \mathcal{O}^{\prime}\right.$ set of $p$ outputs $\}$ is the maximum number of outputs of $W$ in $\mathcal{O}^{\prime}$. This maximum is attained either by choosing all the outputs in $W$ to be in $\mathcal{O}^{\prime}$ if $\operatorname{out}(W) \leq p$, or by choosing $p$ outputs in $W$ to be in $\mathcal{O}^{\prime}$ if $\operatorname{out}(W) \geq p$. Hence, $\max \left\{\right.$ out $^{\prime}(W) \mid \mathcal{O}^{\prime}$ set of $p$ outputs $\}=\min \{p$, out $(W)\}$.

Let $\mathcal{S}_{0}$ (resp. $\mathcal{S}_{1}, \mathcal{S}_{2}$ ) be the set of switches adjacent to no output (resp. one output, two outputs) and $s_{0}$ (resp. $\left.s_{1}, s_{2}\right)$ its cardinality.

Let $\mathcal{S}_{0}^{0}$ (resp. $\mathcal{S}_{0}^{1}, \mathcal{S}_{0}^{2}$ ) be the set of switches of $\mathcal{S}_{0}$ adjacent to no vertex (resp. one vertex, two vertices) of $\mathcal{S}_{2}$ and $s_{0}^{0}$ (resp. $s_{0}^{1}, s_{0}^{2}$ ) its cardinality.

Let $\mathcal{S}_{1}^{0}$ (resp. $\mathcal{S}_{1}^{1}$ ) be the set of switches of $\mathcal{S}_{1}$ adjacent to no vertex (resp. one vertex) of $\mathcal{S}_{2}$ and $s_{1}^{0}\left(\right.$ resp. $s_{1}^{1}$ ) its cardinality.

Let us define the sets $\mathcal{U}_{i}$ and $\mathcal{T}_{i}$ inductively by: $\mathcal{U}_{0}=\mathcal{S}_{1}^{1}$ and $\mathcal{T}_{0}=\mathcal{S}_{1}^{0}$. $\mathcal{U}_{i+1}$ (resp. $\mathcal{T}_{i+1}$ ) is the set of switches of $\mathcal{T}_{i}$ having exactly one (resp. no) neighbour in $\mathcal{U}_{i}$. The cardinality of $\mathcal{U}_{i}$ is denoted $u_{i}$.

Let us denote by $k_{1}^{0}\left(\right.$ resp. $k_{1}^{1}, k_{2}$ ) the number of inputs adjacent to $\mathcal{S}_{1}^{0}$ (resp. $\mathcal{S}_{1}^{1}, \mathcal{S}_{2}$ ).

From Proposition 2, we prove the following assertions:

Proposition 3 1. If $p \geq 2$, a switch is adjacent to at most two outputs. 
2. If $p \geq 3$ then two switches of $\mathcal{S}_{2}$ are not adjacent.

3. If $p \geq 4$, a switch of $\mathcal{S}_{1}$ is adjacent to at most one switch of $\mathcal{S}_{2}$.

4. If $p \geq 5$, a switch of $\mathcal{S}_{0}$ is adjacent to at most two switches of $\mathcal{S}_{2}$.

5. If $p \geq 2 i+4$, then $\left(\mathcal{U}_{i} ; \mathcal{T}_{i}\right)$ is a partition of $\mathcal{T}_{i-1}$.

6. If $p \geq 2 i+5$, then any two elements of $\mathcal{U}_{i}$ are not adjacent.

7. If $p \geq i+6$, then any element of $\mathcal{U}_{i}$ is not adjacent to any element of $\mathcal{S}_{0}^{2}$.

Proof. 1. Suppose that a switch $S$ is adjacent to three outputs. Let $W$ be the set consisting in $S$ and its three adjacent outputs. Then $\operatorname{deg}(W)=1, \operatorname{out}(W)=3$ and $\operatorname{in}(W)=0$. So if $p \geq 3$ then $W$ contradicts Proposition 2 .

2. Suppose that two switches $S$ and $S^{\prime}$ of $\mathcal{S}_{2}$ are adjacent. Let $W$ be the set consisting in $S, S^{\prime}$ and their four adjacent outputs. Then $\operatorname{deg}(W)=2$, out $(W)=4$ and $\operatorname{in}(W)=0$. So if $p \geq 3$ then $W$ contradicts Proposition 2 .

3. Suppose that a switch $S_{1}$ of $\mathcal{S}_{1}$ is adjacent to two switches of $\mathcal{S}_{2}, S_{2}$ and $S_{2}^{\prime}$. Let $W$ be the set consisting in $S_{1}, S_{2}, S_{2}^{\prime}$ and their five adjacent outputs. Then $\operatorname{deg}(W)=3$, $\operatorname{out}(W)=5$ and $i n(W)=0$. So if $p \geq 4$ then $W$ contradicts Proposition 2 .

4. Suppose that a switch $S_{0}$ of $\mathcal{S}_{0}$ is adjacent to three switches of $\mathcal{S}_{2}, S_{2}, S_{2}^{\prime}$ and $S_{2}^{\prime \prime}$. Let $W$ be the set consisting in $S_{0}, S_{2}, S_{2}^{\prime}, S_{2}^{\prime \prime}$ and their six adjacent outputs. Then $\operatorname{deg}(W)=4$, $\operatorname{out}(W)=6$ and $i n(W)=0$. So if $p \geq 5$ then $W$ contradicts Proposition 2 .

5. Suppose that a switch $T$ of $\mathcal{T}_{i-1}$ is adjacent to two switches $U_{i-1}$ and $U_{i-1}^{\prime}$ of $\mathcal{U}_{i-1}$. For $j=2$ to $i$, let $U_{i-j}$ (resp. $U_{i-j}^{\prime}$ ) be the swich of $\mathcal{U}_{i-j}$ adjacent to $U_{i-j+1}$ (resp. $\left.U_{i-j+1}^{\prime}\right)$. Let $S_{2}$ (resp. $S_{2}^{\prime}$ ) be the switch of $\mathcal{S}_{2}$ adjacent to $U_{0}$ (resp. $U_{0}^{\prime}$ ). Let $W$ be the set consisting in $T, U_{j}, 0 \leq j \leq i-1, U_{j}^{\prime}, 0 \leq j \leq i-1, S_{2}, S_{2}^{\prime}$ and their adjacent outputs. Then $\operatorname{deg}(W)=2 i+3, \operatorname{out}(W)=2 i+5$ and $i n(W)=0$. So, if $p \geq 2 i+4$ then $W$ contradicts Proposition 2.

6. Suppose that two switches $U_{i}$ and $U_{i}^{\prime}$ of $\mathcal{U}_{i}$ are adjacent. For $j=1$ to $i$, let $U_{i-j}$ (resp. $U_{i-j}^{\prime}$ ) be the swich of $\mathcal{U}_{i-j}$ adjacent to $U_{i-j+1}\left(\operatorname{resp} . U_{i-j+1}^{\prime}\right)$. Let $S_{2}\left(\operatorname{resp} . S_{2}^{\prime}\right)$ be the switch of $\mathcal{S}_{2}$ adjacent to $U_{0}$ (resp. $U_{0}^{\prime}$ ). Let $W$ be the set consisting in $U_{j}, 0 \leq j \leq i$, $U_{j}^{\prime}, 0 \leq j \leq i, S_{2}, S_{2}^{\prime}$ and their adjacent outputs. Then $\operatorname{deg}(W)=2 i+4$, out $(W)=2 i+6$ and $i n(W)=0$. So, if $p \geq 2 i+5$ then $W$ contradicts Proposition 2 .

7. Suppose that a switch $S_{0}$ of $\mathcal{S}_{0}^{2}$ and a switch $U_{i}$ of $\mathcal{U}_{i}$ are adjacent. For $j=1$ to $i$, let $U_{i-j}$ be the swich of $\mathcal{U}_{i-j}$ adjacent to $U_{i-j+1}$. Let $S_{2}$ be the switch of $\mathcal{S}_{2}$ adjacent to $U_{0}$ and $S_{2}^{\prime}$ and $S_{2}^{\prime \prime}$ be the two vertices of $\mathcal{S}_{2}$ adjacent to $S_{0}$. Let $W$ be the set consisting in $U_{j}, 0 \leq j \leq i, S_{2}, S_{0}, S_{2}^{\prime}$ and $S_{2}^{\prime \prime}$ and their adjacent outputs. Then $\operatorname{deg}(W)=i+5$, $\operatorname{out}(W)=i+7$ and $i n(W)=0$. So, if $p \geq i+6$ then $W$ contradicts Proposition 2 .

From this Proposition, we deduce the following equations: 
Corollary 2

If $p \geq 3$,

$$
\begin{gathered}
n=2 s_{2}+s_{1} \\
2 s_{2} \leq 3 s_{1}+4 s_{0}+p
\end{gathered}
$$

If $p \geq 4$,

$$
2 s_{2} \leq s_{1}+4 s_{0}+p
$$

If $p \geq 5$,

$$
2 s_{2}=s_{1}^{1}+2 s_{0}^{2}+s_{0}^{1}+k_{2}
$$

If $p \geq 2 i+5$,

$$
2 s_{1}^{1}+\sum_{j=1}^{i} u_{j} \leq 3 t_{i}+3 s_{0}^{1}+4 s_{0}^{0}+k_{1}^{1}+k_{1}^{0}
$$

If $p \geq 2 i+6$,

$$
2 s_{1}^{1}+\sum_{j=1}^{i} u_{j} \leq u_{i+1}+3 s_{0}^{1}+4 s_{0}^{0}+k_{1}^{1}+k_{1}^{0}
$$

Proof. Eq. (1) is obtained by counting the number $e$ of edges in $\Delta(\mathcal{O})$. Every output is adjacent to one edge so $e=n$. By Proposition 3, a switch is adjacent to at most two outputs. Then $n=2 s_{2}+s_{1}$.

Eq. (2) and (3) are obtained by counting $e_{0}$ the number of edges between $\mathcal{S}_{2}$ and $\mathcal{S}_{1} \cup$ $\mathcal{S}_{0} \cup \mathcal{I}$. By Proposition 3-2, a switch in $\mathcal{S}_{2}$ is adjacent to exactly two vertices of $\mathcal{S}_{1} \cup \mathcal{S}_{0} \cup \mathcal{I}$. So $e_{0}=2 s_{2}$. A switch of $\mathcal{S}_{0}$ is adjacent to at most 4 switches in $\mathcal{S}_{2}$ and a switch of $\mathcal{S}_{1}$ is adjacent to at most 3 switches in $\mathcal{S}_{2}$ or at most one switch in $\mathcal{S}_{2}$ if $p \geq 4$ by Proposition 3-3. So $e_{0} \leq 3 s_{1}+4 s_{0}+p$ or if $p \geq 4, e_{0} \leq s_{1}+4 s_{0}+p$.

Eq. (4) is also obtained by counting $e_{0}$ : if $p \geq 5$, by Proposition 3-3, $\mathcal{S}_{0}$ is partitionned in $\left(\mathcal{S}_{0}^{2}, \mathcal{S}_{0}^{1}, \mathcal{S}_{0}^{0}\right)$.

Eq. (5) and (6) are obtained by counting the number $e_{i}$ of edges between $\bigcup_{j=0}^{i} \mathcal{U}_{j}$ and $\mathcal{I} \cup \mathcal{S}_{0} \cup \mathcal{T}_{i}$. There are $2 s_{1}^{1}$ edges between $\mathcal{S}_{1}^{1}$ and $\mathcal{S}_{0} \cup \mathcal{S}_{1}$. And by definition of $U_{1}$ and Proposition 3-5, there are $u_{1}$ edges between $\mathcal{S}_{1}^{1}$ and $\mathcal{U}_{1}$ and no between $\mathcal{S}_{1}^{1}$ and $\bigcup_{j=2}^{i} \mathcal{U}_{j}$. So there are $2 s_{1}^{1}-u_{1}$ edges between $\mathcal{S}_{1}^{1}$ and $\mathcal{T}_{i}$. Analogously, for every $1 \leq j<i$, there are $2 u_{j}-u_{j+1}$ edges between $\mathcal{U}_{j}$ and $\mathcal{I} \cup \mathcal{S}_{0} \cup \mathcal{T}_{i}$. If $p \geq 2 i+5$, then by Proposition 3-6, there at $2 u_{i}$ edges between $\mathcal{U}_{i}$ and $\mathcal{I} \cup \mathcal{S}_{0} \cup \mathcal{T}_{i}$. Because there are no edges between $\bigcup_{j=0}^{i} \mathcal{U}_{j}$ and $\mathcal{S}_{0}^{2}$, it follows that $e_{i}=2 s_{1}^{1}+\sum_{j=1}^{i} u_{j}$. Moreover, switches of $\mathcal{T}_{i} \cup \mathcal{S}_{0}^{1}$ are adjacent to at most 3 switches not in $\mathcal{S}_{2}$, switches of $\mathcal{S}_{0}^{0}$ have degree at most 4 and $k_{1}^{1}+k_{0}^{1}$ inputs are adjacent to switches in $\mathcal{S}_{1}$. So $e_{i} \leq 3 t_{i}+3 s_{0}^{1}+4 s_{0}^{0}+k_{1}^{1}+k_{1}^{0}$. If $p \geq 2 i+6$, by Proposition 3-4 the only switches of $\mathcal{T}_{i}$ which are adjacent to vertices of $\bigcup_{j=0}^{i} \mathcal{U}_{j}$ are those of $\mathcal{U}_{i+1}$ which are adjacent to exactly one by definition. So $e_{i} \leq u_{i+1}+3 s_{0}^{1}+4 s_{0}^{0}+k_{1}^{1}+k_{1}^{0}$. 


\section{Theorem 4}
1. If $p \geq 2 p^{\prime}-1, \quad S(p, n) \geq \frac{2^{p^{\prime}+1}-3}{2^{p^{\prime}+1}} n-\frac{2^{p^{\prime}}-3}{2^{p^{\prime}+1}} p$
2. If $p \geq 2 p^{\prime}, \quad S(p, n) \geq \frac{2^{p^{\prime}}-1}{2^{p^{\prime}}} n-\frac{2^{p^{\prime}-1}-1}{2^{p^{\prime}}} p$.

Proof. Since a minimum $(p, n)$-selector must be connected, it follows that $S(p, n) \geq$ $1 / 2(p+n-2)$, hence $S(1, n) \geq\left\lfloor\frac{n}{2}\right\rfloor$ and $S(2, n) \geq\left\lceil\frac{n}{2}\right\rceil$.

If $p \geq 3$, Eq. (1) $+1 / 5$ Eq. (2) gives $8 / 5 s_{2}+8 / 5 s_{1}+4 / 5 s_{0} \geq n-\frac{3}{5}$. Thus $S(3, n) \geq \frac{5 n}{8}-\frac{3}{8}$.

If $p \geq 4$, Eq. (1) $+\frac{1}{3}$ Eq. (3) gives $4 / 3 s_{0}+4 / 3 s_{1}+4 / 3 s_{2} \geq n-4 / 3$. Thus $S(4, n) \geq \frac{3 n}{4}-1$.

Suppose now that $p \geq 5$.

1) Set $l=p^{\prime}-3$.

$$
\begin{aligned}
& \text { Eq. (1) }+\frac{1}{2^{l+4}-3}\left\{\left(2^{l+3}-3\right) \text { Eq. (4) }+\sum_{i=0}^{l-1} 2^{l+1-i} \text { Eq. (6) }[i]+\text { Eq. (5) }[l]\right\} \text { yields: } \\
& \qquad \leq \frac{2^{l+4}}{2^{l+4}-3}\left(s_{2}+s_{1}^{1}+\sum_{i=1}^{l} u_{i}+t_{l}\right)+\frac{2^{l+4}-6}{2^{l+4}-3} s_{0}^{2}+\frac{7 \times 2^{l+1}-12}{2^{l+4}-3} s_{0}^{1}+\frac{2^{l+4}-12}{2^{l+4}-3} s_{0}^{0} \\
& \quad+\frac{2^{l+3}-3}{2^{l+4}-3} k_{2}+\frac{2^{l+2}-4}{2^{l+4}-3} k_{1}^{1}+\frac{2^{l+1}-4}{2^{l+4}-3} k_{1}^{0}
\end{aligned}
$$

Thus $n \leq \frac{2^{l+4}}{2^{l+4}-3} s+\frac{2^{l+3}-3}{2^{l+4}-3} p$.

2) Set $l=p^{\prime}-3$.

$$
\begin{aligned}
& \text { Eq. (1) }+\frac{1}{2^{l+3}-1}\left\{\left(2^{l+2}-1\right) \text { Eq. (4) }+\sum_{i=0}^{l} 2^{l-i} \text { Eq. (6) }[i]\right\} \text { yields: } \\
& \qquad \leq \frac{2^{l+3}}{2^{l+3}-1}\left(s_{2}+s_{1}^{1}+\sum_{i=1}^{l+1} u_{i}\right)+t_{l+1}+\frac{2^{l+3}-2}{2^{l+3}-1} s_{0}^{2}+\frac{7 \times 2^{l}-2}{2^{l+3}-1} s_{0}^{1}+\frac{2^{l+3}-4}{2^{l+3}-1} s_{0}^{0} \\
& \quad+\frac{2^{l+2}-1}{2^{l+3}-1} k_{2}+\frac{2^{l+1}-1}{2^{l+3}-1} k_{1}^{1}+\frac{2^{l}-1}{2^{l+3}-1} k_{1}^{0}
\end{aligned}
$$

Thus $n \leq \frac{2^{l+3}}{2^{l+3}-1} s+\frac{2^{l+2}-1}{2^{l+3}-1} p$.

We conjecture that the inequalities obtained in the above corollary are tight:

Conjecture 1 Let $p$ be a fixed non-negative integer,

if $p$ is even then $S(p, n)=\frac{2^{p / 2}-1}{2^{p / 2}} n+\Theta(1)$;

if $p$ is odd then $S(p, n)=\frac{2^{(p+3) / 2}-3}{2^{(p+3) / 2}} n+\Theta(1)$. 


\subsection{Minimum $(p, n)$-selectors for $p$ fixed}

We now show that Conjecture 1 holds for $p \leq 6$.

Therefore we prove a reinforcement of Proposition 2, which allows us to check the cut criterion only for a certain kind of subsets called suitable. A subset is suitable if it is connected, with no input and containing all the outputs adjacent to its switches.

Proposition $4 A(p, n)$-network is a $(p, n)$-selector if and only if $\operatorname{deg}(W) \geq \min \{p$, out $(W)\}$ for any suitable subset $W$.

Proof. Suppose that $\operatorname{deg}(W) \geq \min \{p, \operatorname{out}(W)\}$ for any suitable subset $W$.

Let us first prove that for any subset $X$ connected with no input then $\operatorname{deg}(X) \geq$ $\min \{p$, out $(X)\}$. Let $W$ be the set obtained from $X$ by adding all the outputs adjacent to a switch of $X$. Then $\operatorname{deg}(W) \leq \operatorname{deg}(X)$ and $\operatorname{out}(W) \geq \operatorname{out}(X)$. So $\operatorname{deg}(X) \geq \operatorname{deg}(W) \geq$ $\min \{p$, out $(W)\} \geq \min \{p$, out $(X)\}$.

Let us prove that for any subset $Y$ with no input then $\operatorname{deg}(Y) \geq \min \{p, \operatorname{out}(Y)\}$, by induction on the number $c$ of connected component. The result is true if $c=1$. Suppose now that it is true for $c$, and suppose $Y$ has $c+1$ connected components. Let $C$ be one of these and $X=Y \backslash C$. We have $\operatorname{deg}(Y)=\operatorname{deg}(C)+\operatorname{deg}(X) \geq \min \{p, \operatorname{out}(C)\}+\min \{p, \operatorname{out}(X)\}$. Since $\operatorname{out}(Y)=\operatorname{out}(C)+\operatorname{out}(X)$, we obtain $\operatorname{deg}(Y) \geq \min \{p, \operatorname{out}(Y)\}$.

Let us now prove that for any subset $Z, \operatorname{deg}(Z) \geq \min \{p, \operatorname{out}(Z)\}-i n(Z)$. Let $Y$ be the set obtained from $Z$ by removing all the inputs. We have $\operatorname{deg}(Y) \geq \operatorname{deg}(Z)-i n(Z)$, and $\operatorname{out}(Y)=\operatorname{out}(Z)$. Now $\operatorname{deg}(Y) \geq \min \{p, \operatorname{out}(Y)\}$, so $\operatorname{deg}(Z) \geq \min \{p, \operatorname{out}(Z)\}-\operatorname{in}(Z)$.

\section{Theorem 5}

$$
\begin{aligned}
& S(1, n)=\left\lfloor\frac{n}{2}\right\rfloor \\
& S(2, n)=\left\lceil\frac{n}{2}\right\rceil
\end{aligned}
$$

Proof. Let $P_{i}$ be the network consisting of a path $\left(v_{1}, v_{2}, \ldots, v_{i}\right)$ of switches and $2 i$ outputs $1 \leq o_{j} \leq 2 i$ such that for every $1 \leq j \leq i$, then $v_{j}$ is adjacent to $o_{j}$ and $o_{i+j}$. Let $S_{1,2 i+1}$ (resp. $S_{2,2 i}$ ) be the network obtained from $P_{i}$ by adding an input adjacent to $v_{1}$ and an output (resp. an input) adjacent to $v_{i}$ see Figure 5 .

Let $W$ be a suitable subset of $S_{1,2 i+1}$. And let $j$ be the smallest integer such that $v_{j} \in W$. Then $v_{i}$ is adjacent to an element in $\bar{W}$. Thus $\operatorname{deg}(W) \geq 1$. By Proposition 4, it follows that $S_{1,2 i+1}$ is a $(1,2 i+1)$-selector.

Analogously considering $j$ and $j^{\prime}$ the smallest and largest integer such that $v_{j}$ is in a suitable subset $W$ of $S_{2,2 i}$, we obtain that $\operatorname{deg}(W) \geq 2$ for any suitable subset of $S_{2,2 i}$. Hence $S_{2,2 i}$ is a $(2,2 i)$-selector by Proposition 4 .

The network $S_{1,2 i}$ (resp. $S_{2,2 i-1}$ ) obtained from $S_{1,2 i+1}$ (resp. $S_{2,2 i}$ ) by removing an output is obviously a $(1,2 i)$-selector (resp. $(2,2 i-1)$-selector). 


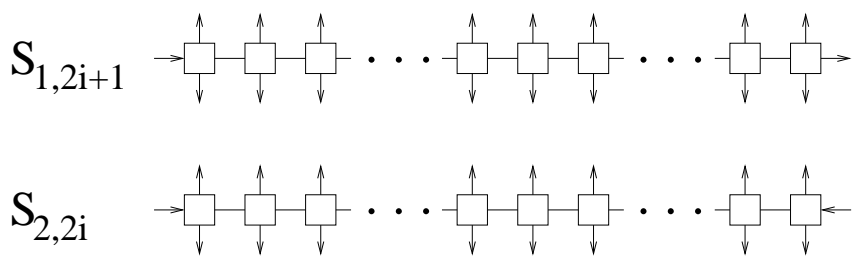

Figure 5: Minimum $(1,2 i+1)$ - and $(2,2 i)$-selectors.

\section{Theorem 6}

$$
S(3, n)=\left\lceil\frac{5 n}{8}\right\rceil+\Theta(1)
$$

Proof. Let $S_{3,8 i+5}$ be the network depicted Figure 6 . Let $W$ be a suitable subset of $S_{3,8 i+5}$.

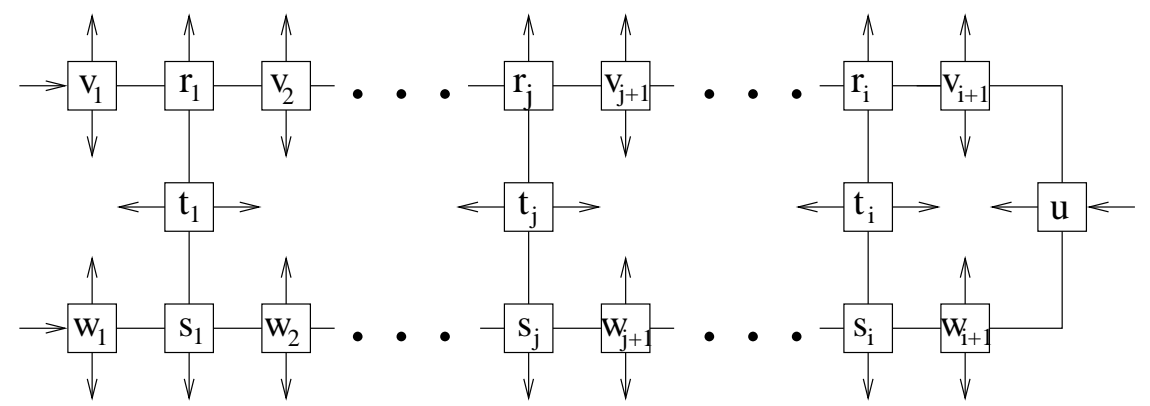

Figure 6: Minimum $(3, n)$-selector

If $W$ contains a unique switch then $\operatorname{deg}(W) \geq 2 \geq \operatorname{out}(W)$. Set $u=r_{i+1}=s_{i+1}$ and let $R=\left\{r_{j}, 1 \leq j \leq i+1\right\}$ and $S=\left\{r_{j}, 1 \leq j \leq i+1\right\}$. Suppose now that $W$ contains at least two switches. Then because $W$ is connected, it contains an element of $R \cup S$. By symmetry, we may assume that $W \cap R$ is not empty. Let $j$ and $j^{\prime}$ be the smallest and largest integer such that $r_{j} \in W$. Then if $v_{j} \in \bar{W}$ then $v_{j} r_{j} \in \Delta(W)$ otherwise $v_{j} r_{j-1}$ (with $r_{0}$ being the input adjacent to $v_{1}$ ) is in $\Delta(W)$. Analogously, if $v_{j^{\prime}+1} \in \bar{W}$ then $v_{j^{\prime}+1} r_{j^{\prime}} \in \Delta(W)$ otherwise $v_{j^{\prime}+1} r_{j^{\prime}+2}$ (with $r_{i+2}$ being the input adjacent to $u$ ) is in $\Delta(W)$.

Suppose first that $W \cap S \neq \emptyset$. Let $j^{\prime \prime}$ be the smallest integer such that $s_{j^{\prime \prime}} \in W$. There is a cutting edge which is incident to $w_{j^{\prime \prime}}$. Hence $\operatorname{deg}(W) \geq 3$.

Suppose now that $W \cap S=\emptyset$. Then $j \leq i$ and $r_{j} t_{j}$ or $t_{j} s_{j}$ is in $\Delta(W)$. Again $\operatorname{deg}(W) \geq 3$.

Thus by Proposition $4, S_{3,8 i+5}$ is a $(3,8 i+5)$-selector. Obviously, for $1 \leq j \leq 7, S_{3,8 i+5-j}$ obtained from $S_{3,8 i+5}$ by removing $j$ outputs is a $(3,8 i+5-j)$-selector. 


\section{Theorem 7}

$$
S(4, n)=\frac{3}{4} n+\Theta(1)
$$

Proof. Let $S_{4,4 i}$ be the network depicted Figure 7 .

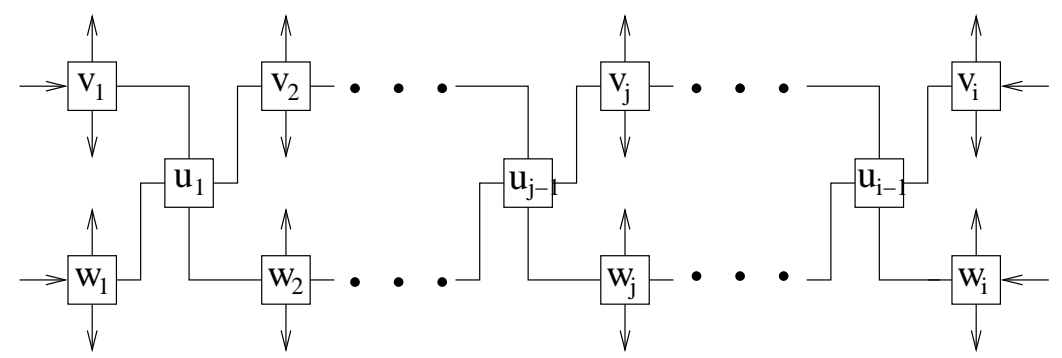

Figure 7: Minimum $(4, n)$-selector

Let $W$ be a suitable subset of $S_{4,4 i}$. If $W$ contains a unique switch then $\operatorname{deg}(W) \geq 2 \geq$ $\operatorname{out}(W)$. Suppose now that it contains at least two switches. Then because $W$ is connected, it contains at least one of the $u_{j}$. Let $j$ and $j^{\prime}$ be the smallest and largest integer such that $u_{j}, u_{j^{\prime}} \in W$. Then one of the two edges $v_{j} u_{j}$ and $v_{j} u_{j-1}$ is in $\Delta(W)$ since $u_{j-1} \notin W$ (with $u_{0}$ the input adjacent to $\left.v_{1}\right)$. Analogously $w_{j}, v_{j^{\prime}+1}$ and $w_{j^{\prime}+1}$ are incident to a $W$-cutting edge. Hence $\operatorname{deg}(W) \geq 4$. Therefore, by Proposition $4, S_{4,4 i}$ is a $(4,4 i)$-selector.

Obviously, for $1 \leq j \leq 3$, the network $S_{4,4 i-j}$, obtained from $S_{4,4 i}$ by removing $j$ outputs is a $(4,4 i-j)$-selector.

\section{Theorem 8}

$$
S(5, n)=\frac{13}{16} n+\Theta(1)
$$

Proof. It is simple matter to check that the network $S_{5,16 i}$ depicted Figure 8 is a $(5,16 i)$ selector. For $1 \leq j \leq 15$, the network $S_{5,16 i-j}$, obtained from $S_{5,16 i}$ by removing $j$ outputs is a $(5,16 i-j)$-selector.

\section{Theorem 9}

$$
S(6, n)=\frac{7}{8} n+\Theta(1)
$$

Proof. Let $S_{6,8 i}$ be the network depicted Figure 9. Set $P_{A}=\left(a_{1}, a_{2}, \ldots, a_{i}\right), P_{B}=$ $\left(b_{1}, b_{2}, \ldots, b_{i}\right), P_{C}=\left(c_{1}, c_{2}, \ldots, c_{i}\right)$ and $P_{D}=\left(d_{1}, d_{2}, \ldots, d_{i}\right)$. Let $W$ be a suitable set of $S_{6,8 i}$. 


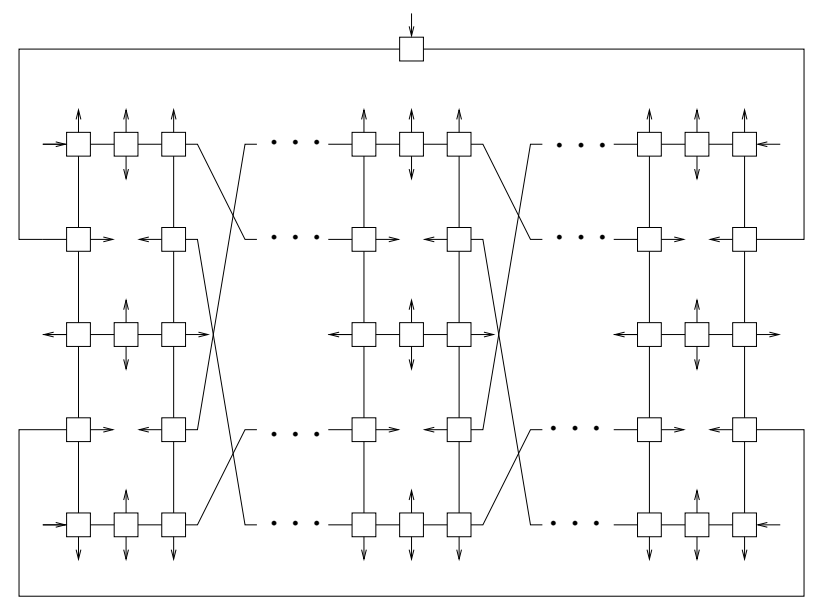

Figure 8: Minimum $(5, n)$-selector

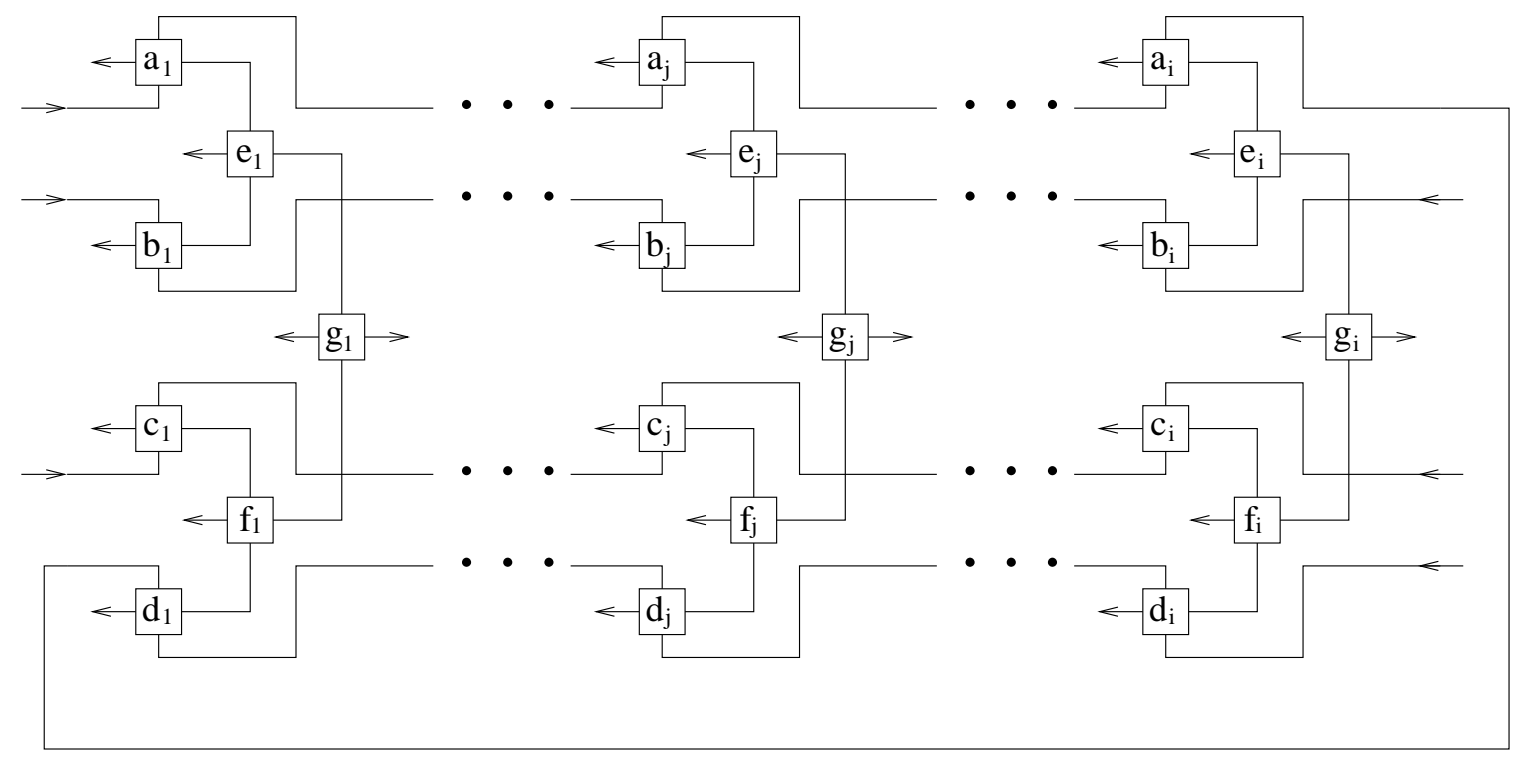

Figure 9: Minimum $(6, n)$-selector 
Assume first that $W$ has $s w<6$ switches. Since there is no cycle of length less than 6 and the distance between to switches of $G$ is at least 6 , then $W$ is a tree containing at most one element of $G$. Thus, $\operatorname{deg}(W) \geq 2 s w+2-\operatorname{out}(W)$ and $\operatorname{out}(W) \leq s w+1$. Thus, $\operatorname{deg}(W) \geq \operatorname{out}(W)$.

Suppose now that $W$ has at least 6 switches. Let us prove that $\operatorname{deg}(W) \geq 6$.

Let us consider the paths $P_{1}=P_{A} P_{D}, P_{2}=P_{B}$ and $P_{3}=P_{C}$. For $1 \leq l \leq 3$, if there is a vertex on $P_{l}$ then $P_{l}$ contains at least two cutting edges. In particular, if there is a vertex on each path then $\operatorname{deg}(W) \geq 6$.

Suppose now that $W$ intersects two paths $P_{l}$. Let $T_{j}$ be the network induced by $\left\{a_{j}, b_{j}, c_{j}, d_{j}, e_{j}, f_{j}, g_{j}\right\}$. Then each $T_{j}$ containing a vertex of $W$ contains a $W$-cutting edge. If there at least two such trees, then $\operatorname{deg}(W) \geq 6$ because there are at least four cutting edges on the paths. If there only one, it is easy that there are six cutting edges because there are 4 on $P_{1}$, (two on $P_{A}$ and two on $P_{D}$ ). So $\operatorname{deg}(W) \geq 6$.

At last, assume that $W$ intersects one of the $P_{l}$. For any tree $T_{j}$, if $\left|W \cap T_{j}\right|=1$, there is one cutting edge in $T_{j}$, if $\left|W \cap T_{j}\right| \in\{2,3\}$ there are two cutting edges and if $\left|W \cap T_{j}\right|=4$, there are three cutting edges. It follows easily that $\operatorname{deg}(W) \geq 6$.

\subsection{Lower bounds for superselectors}

Proposition 5 A minimum n-superselector is connected.

Corollary $3 S^{+}(n) \geq n-1$.

Proof. Since the switches have degree at most four and inputs and outputs degree one, denoting by $e$ the number of edges connecting two switches, we obtain : $4 s w \geq 2 e+2 n$. Since a minimum superselector is connected $e \geq s w-1$. It follows $2 s w \geq 2 n-2$.

Proposition 6 Let $G=(V, E)$ be an n-selector and $W$ a subset of vertices such that $\operatorname{out}(W)+i n(W) \leq n$. Then $\operatorname{deg}(W) \geq \max \{\operatorname{out}(W), i n(W)\} \geq \frac{\operatorname{out}(W)+i n(W)}{2}$.

Proof. Let $W$ be a set such that $\operatorname{out}(W)+i n(W) \leq n$. Set $\mathcal{O}^{\prime}=\operatorname{out}(W)$ and $\mathcal{I}^{\prime}$ be a set of $\left|\mathcal{O}^{\prime}\right|$ inputs in $\mathcal{I} \backslash i n(W)$. Such a set $\mathcal{I}^{\prime}$ exists since $\operatorname{out}(W)+i n(W) \leq n$. A variant of the Ford-Fulkerson Theorem states that the problem is feasible only if

$$
\operatorname{deg}(W) \geq \operatorname{demand}(W)=\mathcal{O}^{\prime} \cap W-\mathcal{I}^{\prime} \cap W=\operatorname{out}(W)
$$

Analgously, setting $\mathcal{I}^{\prime}=i n(W)$ and $\mathcal{O}^{\prime}$ be a set of $\left|\mathcal{I}^{\prime}\right|$ outputs in $\mathcal{O} \backslash$ out $(W)$, we get $\operatorname{deg}(W) \geq \operatorname{in}(W)$.

Proposition 7 If $n \geq 3$ then a switch is adjacent to at most two elements of $\mathcal{I} \cup \mathcal{O}$. 
Proof. Suppose a vertex $S$ is adjacent to at least three outputs or inputs. Then $W$ the union of $S$ and its adjacent inputs and outputs. Then $\operatorname{deg}(W)=1$ and $i n(W)+o u t(W)=3$. So if $n \geq 3$ then $W$ contradicts Proposition 6 .

Since there are $n$ inputs and $n$ outputs in an $n$-superselector we get the following :

Corollary 4 If $n \geq 3$ then $S^{+}(n) \geq n$.

Definition 2 Let $\mathcal{V}_{0}$ (resp. $\mathcal{V}_{1}, \mathcal{V}_{2}$ ) be the set of switches adjacent to no (resp. one, two) elements of $\mathcal{I} \cup \mathcal{O}$ and $v_{0}\left(\right.$ resp. $\left.v_{1}, v_{2}\right)$ its cardinality. By Proposition $7,\left(\mathcal{V}_{0}, \mathcal{V}_{1}, \mathcal{V}_{2}\right)$ is a partition of $\mathcal{V}$ thus $s w=v_{0}+v_{1}+v_{2}$.

Since there are $n$ outputs and $n$ inputs then

$$
2 n=2 v_{2}+v_{1}
$$

Assume now that $n \geq 6$.

Proposition 8 If $n \geq 6$, an element of $\mathcal{V}_{2}$ is adjacent to at most one element of $\mathcal{V}_{2}$

Proof. Suppose that a swicth $V_{2}$ of $\mathcal{V}_{2}$ is adjacent to two switches $V_{2}^{\prime}$ and $V_{2}^{\prime \prime}$ of $\mathcal{V}_{2}$. Let $W$ be the union of $V_{2}, V_{2}^{\prime} V_{2}^{\prime \prime}$ and their adjacent inputs and outputs. Then $\operatorname{deg}(W)=2$ and out $(W)+i n(W)=6$. Hence if $n \geq 6, W$ contradicts Proposition 6 .

Corollary 5 If $n \geq 6$,

$$
v_{2} \leq 3 v_{1}+4 v_{0}
$$

Proof. Let us count the number $e_{2}$ of edges between $\mathcal{V}_{2}$ and $\mathcal{V}_{1} \cup \mathcal{V}_{0}$. By Proposition 8, every switch of $\mathcal{V}_{2}$ is adjacent to at least one switch in $\mathcal{V}_{1} \cup \mathcal{V}_{0}$. So $e_{2} \geq v_{2}$. A switch of $\mathcal{V}_{1}$ $\left(\right.$ resp. $\mathcal{V}_{0}$ ) is adjacent to at most 3 (resp. at most 4) switches, so $e_{2} \leq 3 v_{1}+4 v_{0}$.

Corollary 6 If $n \geq 6$,

$$
S^{+}(n) \geq \frac{8}{7} n
$$

Proof. Eq. (9) $+1 / 4$ Eq. (10) yields: $2 n \leq 7 / 4 v_{2}+7 / 4 v_{1}+v_{0}$. Thus $s w \geq 8 / 7 n$.

Suppose now that $n \geq 7$.

Proposition 9 If $n \geq 7$, an element of $\mathcal{V}_{1}$ is adjacent to at most two elements of $\mathcal{V}_{2}$ 
Proof. Suppose that a switch $V_{1}$ of $\mathcal{V}_{1}$ is adjacent to three switches $V_{2}, V_{2}$ and $V_{2}^{\prime \prime}$ of $\mathcal{V}_{2}$. Let $W$ be the union of $V_{1}, V_{2}, V_{2}^{\prime} V_{2}^{\prime \prime}$ and their adjacent inputs and outputs. Then $\operatorname{deg}(W)=3$ and $\operatorname{out}(W)+i n(W)=7$. Hence if $n \geq 7, W$ contradicts Proposition 6 .

Let $\mathcal{V}_{2}^{\prime}$ (resp. $\mathcal{V}_{2}^{\prime \prime}$ be the set of switches of $\mathcal{V}_{2}$ which are adjacent to one (resp. no) switch of $\mathcal{V}_{2}$, and $v_{2}^{\prime}$ (resp. $v_{2}^{\prime \prime}$ ) its cardinality. By Proposition $8, \mathcal{V}_{2}^{\prime} \cup \mathcal{V}_{2}^{\prime \prime}=\mathcal{V}_{2}$. So $v_{2}=v_{2}^{\prime}+v_{2}^{\prime \prime}$.

For $i=0,1,2$, let $\mathcal{V}_{1}^{i}$ be the set of switches of $\mathcal{V}_{1}$ which are adjacent to $i$ switch of $\mathcal{V}_{2}$ and $v_{1}^{i}$ its cardinality. By Proposition $9, \mathcal{V}_{1}^{2} \cup \mathcal{V}_{1}^{1} \cup \mathcal{V}_{1}^{0}=\mathcal{V}_{1}$, so $v_{1}=v_{1}^{2}+v_{1}^{1}+v_{1}^{0}$.

Hence Eq. (9) and (10) become

$$
2 n=2 v_{2}^{\prime}+2 v_{2}^{\prime \prime}+v_{1}^{2}+v_{1}^{1}+v_{1}^{0}
$$

Corollary 7 If $n \geq 7$,

$$
v_{2}^{\prime}+2 v_{2}^{\prime \prime} \leq 2 v_{1}^{2}+v_{1}^{1}+4 v_{0}
$$

Proof. By counting $e_{2}$ the number of edges between $\mathcal{V}_{2}$ and $\mathcal{V}_{1} \cup \mathcal{V}_{0}$. The result follows from the definitions of $\mathcal{V}_{2}^{\prime}, \mathcal{V}_{2}^{\prime \prime}$ and $\mathcal{V}_{1}^{i}, 0 \leq i \leq 2$ and the fact that a switch of $\mathcal{V}_{0}$ has degree at most 4 .

Proposition 10 If $n \geq 7$, a switch of $\mathcal{V}_{2}^{\prime}$ is not adjacent to a switch of $\mathcal{V}_{1}^{2}$.

Proof. Suppose that a switch $V_{2}^{\prime}$ of $\mathcal{V}_{2}^{\prime}$ is adjacent to a switch $V_{1}^{2}$ of $\mathcal{V}_{1}^{2}$. Let $W_{2}^{\prime}$ be the switch of $\mathcal{V}_{2}^{\prime}$ adjacent to $V_{2}^{\prime}$ and $T_{2}^{\prime}$ the switch of $\mathcal{V}_{2}$ distinct from $V_{2}^{\prime}$ adjacent to $V_{1}^{2}$. Let $W$ be the union of $V_{2}^{\prime}, W_{2}^{\prime}, T_{2}^{\prime} V_{1}^{2}$ and their adjacent inputs and outputs. Then $\operatorname{deg}(W)=3$ and $\operatorname{out}(W)+i n(W)=7$. Hence if $n \geq 7, W$ contradicts Proposition 6 .

Corollary 8 If $n \geq 7$,

$$
v_{2}^{\prime} \leq v_{1}^{1}+4 v_{0}
$$

Corollary 9 If $n \geq 7$,

$$
S^{+}(n) \geq \frac{5}{4} n
$$

Proof. Eq. (11) $+1 / 5$ Eq. (12) $+1 / 5$ Eq. (13) yields: $2 n \leq 8 / 5\left(v_{2}^{\prime}+v_{2}^{\prime \prime}+v_{0}+7 / 5\left(v_{1}^{2}+\right.\right.$ $\left.v_{1}^{1}\right)+v_{1}^{0}$. Thus $s w \geq 5 / 4 n$.

Suppose now that $n \geq 10$.

Proposition 11 If $n \geq 10$ then an element of $\mathcal{V}_{0}$ is adjacent to at most two elements of $\mathcal{V}_{2}^{\prime}$.

Proof. Suppose that a switch $V_{0}$ of $\mathcal{V}_{0}$ is adjacent to three switches $V_{2}, W_{2}, T_{2}$ of $\mathcal{V}_{2}^{\prime}$. Let $V_{2}^{\prime}$, (resp. $W_{2}^{\prime}$ ) be the neighbour of $V_{2}$ (resp. $W_{2}$ ) in $\mathcal{V}_{2}^{\prime}$. Let $W$ be the set consisting in $V_{0}, V_{2}, V_{2}^{\prime}$, $W_{2}, W_{2}^{\prime}, T_{2}^{\prime}$ and their adjacent inputs and outputs. $\operatorname{deg}(W)=4$ and $i n(W)+\operatorname{out}(W)=10$. So if $n \geq 10$, then $W$ contradicts Proposition 6 . 
Corollary 10 If $n \geq 10$

$$
v_{2}^{\prime} \leq v_{1}^{1}+2 v_{0}
$$

Corollary 11 If $n \geq 10$,

$$
S^{+}(n) \geq \frac{4}{3} n
$$

Proof. Eq. (11) $+1 / 4$ Eq. (12) $+1 / 4$ Eq. (14) yields: $2 n \leq 3 / 2\left(v_{2}^{\prime}+v_{2}^{\prime \prime}+v_{0}+v_{1}^{2}+v_{1}^{1}\right)+v_{1}^{0}$. Thus $s w \geq 4 / 3 n$.

For $i=0,1,2$, let $\mathcal{V}_{0}^{i}$ be the set of switches of $\mathcal{V}_{0}$ which are adjacent to $i$ switch of $\mathcal{V}_{2}^{\prime}$ and $v_{0}^{i}$ its cardinality. By Proposition 11, $\mathcal{V}_{0}^{2} \cup \mathcal{V}_{0}^{1} \cup \mathcal{V}_{0}^{0}=\mathcal{V}_{0}$, so $v_{0}=v_{0}^{2}+v_{0}^{1}+v_{0}^{0}$.

Proposition 12 If $n \geq 10$, two elements of $\mathcal{V}_{1}^{2}$ are not adjacent.

Proof. Suppose that a switch $S$ of $\mathcal{V}_{1}^{2}$ is adjacent to a switch $S^{\prime}$ of $\mathcal{V}_{1}^{2}$. Let $V_{2}$ and $W_{2}$ be the two neighbours of $S$ in $\mathcal{V}_{2}$ and $V_{2}^{\prime}$ and $W_{2}^{\prime}$ be the two neighbours of $S^{\prime}$ in $\mathcal{V}_{2}$. Let $W$ be the set consisting in $S, V_{2}, V_{2}^{\prime}, S^{\prime}, V_{2}^{\prime}, W_{2}^{\prime}$ and their adjacent inputs and outputs. $\operatorname{deg}(W)=4$ and $i n(W)+\operatorname{out}(W)=10$. So if $n \geq 10$, then $W$ contradicts Proposition 6 .

\subsection{Minimum $n$-superselectors}

\section{Proposition 13}

i) $S^{+}(1)=0$

ii) $S^{+}(2)=1$

Proof.

i) Obvious.

ii) The network $S_{2}$ consisting of a switch adjacent to two inputs and two outputs is a 2-superselector. Thus $S^{+}(2) \leq 1$. And by Corollary $3 S^{+}(2) \geq 1$.

The following lemma immediatly follows from the definition of superselector:

Lemma 4 Let $G$ be an $n$-superselector with a switch $s$ adjacent to an input $i$, an ouput o and two switches $s_{1}$ and $s_{2}$. Then the network $G^{\prime}$ obtained by removing $s, i$, o and adding the link $s_{1} s_{2}$ is an $(n-1)$-superselector.

Proposition 14 If $3 \leq n \leq 5$ then $S^{+}(n)=n$. 

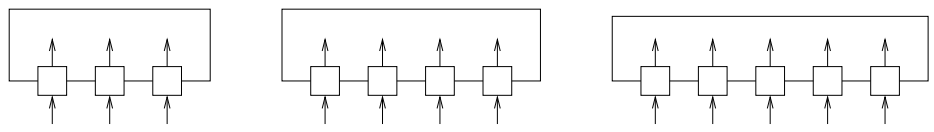

Figure 10: Minimum $n$-superselector for $3 \leq n \leq 5$

Proof. By Corollary $4, S^{+}(n) \geq n$, if $n \geq 3$.

For $3 \leq n \leq 5$, let $S_{n}$ be the network consisting of a cycle of n switches $s_{j}, 1 \leq j \leq n$ each of which is adjacent to an input $i_{j}$ and an output $o_{j}$. (see Figure 10). Let us prove that $S_{5}$ is a 5 -superselector. Let $\mathcal{I}^{\prime}$ and $\mathcal{O}^{\prime}$ be subset of $\mathcal{I}$, and $\mathcal{O}$ respectively such that $\left|\mathcal{I}^{\prime}\right|=\left|\mathcal{O}^{\prime}\right|=k \leq 5$. If the input $i_{j}$ and the output $o_{j}$ are in $\mathcal{I}^{\prime}$ and $\mathcal{O}^{\prime}$ then route $i_{j}$ to $o_{j}$ directly through $s_{j}$. It remains at most two inputs to route and one can do it using the cycle.

By Lemma $4, S_{4}$ and $S_{3}$ are 4 - and 3-superselectors.

Proposition $15 S^{+}(6)=7$

Proof. By Corollary $6, S^{+}(6) \geq 48 / 7$ so $S^{+}(6) \geq 7$. Now it is simple matter to check that the network depicted Figure 11 is a 6 -superselector.

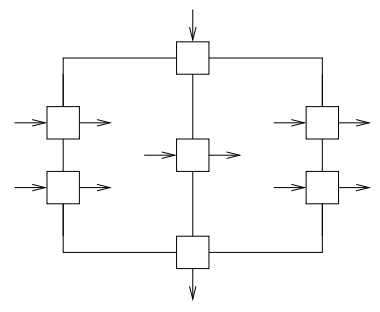

Figure 11: Minimum 6-superselector

Proposition $16 S^{+}(7)=9$ and $S^{+}(8)=10$.

Proof. By Corollary $9, S^{+}(7) \geq 35 / 4$, so $S^{+}(7) \geq 9$ and $S^{+}(8) \geq 10$.

Let us prove that $S_{8}$, the network depicted Figure 12 right, is a 8-superselector. For $1 \leq j \leq 8$, set $P_{j}=\left(i_{j}, s_{j}, o_{j}\right)$ and $Q_{j}=\left(i_{j}, s_{j}, s_{j+4}, o_{j+4}\right) . \quad(j+4$ must be understood modulo 8). Let $\mathcal{I}^{\prime}$ and $\mathcal{O}^{\prime}$ be sets of $k$ inputs and $k$ outputs of $S_{8}$. If $i_{j} \in \mathcal{I}^{\prime}$ and $o_{j} \in \mathcal{O}^{\prime}$ then route $i_{j}$ to $o_{j}$ through $P_{j}$. Let $\mathcal{I}^{\prime \prime}=\left\{i_{j} \in \mathcal{I}^{\prime}, o_{j} \notin \mathcal{O}^{\prime}\right\}$ and $\mathcal{O}^{\prime \prime}=\left\{o_{j} \in \mathcal{O}^{\prime}, i_{j} \notin \mathcal{I}^{\prime}\right\}$. It remains to route inputs of $\mathcal{I}^{\prime \prime}$ to outputs of $\mathcal{O}^{\prime \prime}$ and obviously $\left|\mathcal{I}^{\prime \prime}\right|=\left|\mathcal{O}^{\prime \prime}\right| \leq 4$. If $i_{j} \in \mathcal{I}^{\prime \prime}$ 

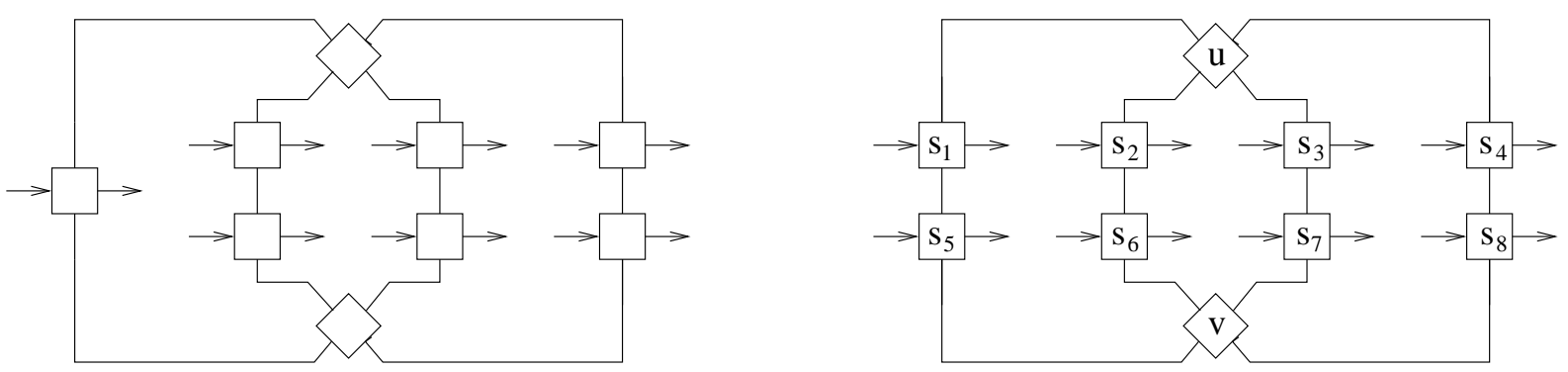

Figure 12: Minimum 7- and 8-superselector

and $o_{j+4} \in \mathcal{O}^{\prime \prime}$ then route $i_{j}$ to $o_{j+4}$ through $Q_{j}$. Let $\mathcal{I}^{3}=\left\{i_{j} \in \mathcal{I}^{\prime}, o_{j} \notin \mathcal{O}^{\prime}\right.$ and $\left.o_{j+4} \notin \mathcal{O}^{\prime}\right\}$ and $\mathcal{O}^{\prime \prime}=\left\{o_{j} \in \mathcal{O}^{\prime}, i_{j} \notin \mathcal{I}^{\prime}\right\}$ and $\left.i_{j+4} \notin \mathcal{I}^{\prime}\right\}$. It remains to route inputs of $\mathcal{I}^{3}$ to outputs of $\mathcal{O}^{3}$ and obviously $\left|\mathcal{I}^{3}\right|=\left|\mathcal{O}^{3}\right| \leq 4$. Hence this can be done through $u$ and $v$.

By Proposition 4, the network depicted Figure 12 left is a 7 -superselector.

\section{Proposition 17}

$$
S^{+}(9)=12
$$

Proof. By Corollary $9, S^{+}(9) \geq 45 / 4$, so $S^{+}(9) \geq 12$.

Let us prove that $S_{9}$, the network depicted Figure 13, is a 9-superselector. For $1 \leq j \leq 6$,

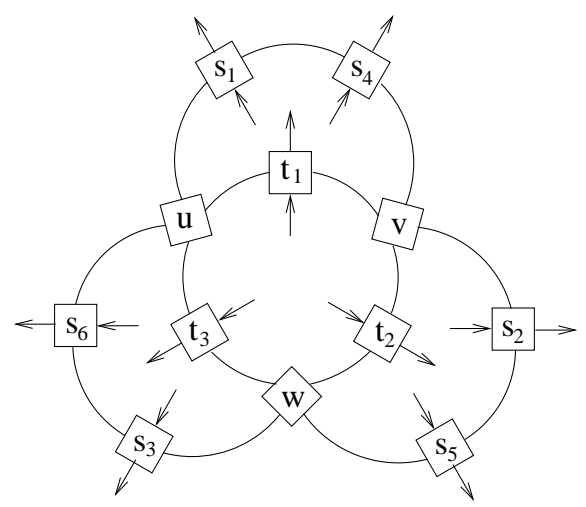

Figure 13: Minimum 9-superselector

set $P_{j}=\left(i_{j}, s_{j}, o_{j}\right), Q_{j}=\left(i_{j}, s_{j}, s_{j+3}, o_{j+3}\right) .(j+3$ must be understood modulo 6$)$ and for $7 \leq j \leq 9$, set $P_{j}=\left(i_{j}, t_{j-6}, o_{j}\right)$. 
Let $\mathcal{I}^{\prime}$ and $\mathcal{O}^{\prime}$ be sets of $k$ inputs and $k$ outputs of $S_{9}$. If $i_{j} \in \mathcal{I}^{\prime}$ and $o_{j} \in \mathcal{O}^{\prime}$ then route $i_{j}$ to $o_{j}$ through $P_{j}$. It remains then at most 4 inputs to route to four outputs. If $i_{j} \in \mathcal{I}^{\prime}$, $o_{j} \notin \mathcal{O}^{\prime}, i_{j+3} \notin \mathcal{I}^{\prime}$ and $o_{j+3[\bmod 6]} \in \mathcal{O}^{\prime}$ then route $i_{j}$ to $o_{j+3}$ through $Q_{j}$. It is now easy to see that we may route the remaining inputs to the remaining outputs through $u, v$ and $w$.

\section{Conclusion and open problems}

In Section 2.4, we obtain linear upper bounds for $R(n, p, f)$. However these bounds are far to be satisfactory because they seems far to be optimal and also because their variations are "opposite" to the variations of $R(n, p, f)$. Indeed, according to the value of $R(n, p, f)$ for small $n, p$ and $f$ and the fact that $R(n, p, f)=R(n, n-p, f)$, we conjecture that as a function of $p, R(n, p, f)$ is unimodal and maximum for $p=n / 2$.

\section{Conjecture 2}

If $p \leq n / 2$ then $R(n, p-1,0) \leq R(n, p, 0)$.

If $p \geq n / 2$ then $R(n, p, 0) \leq R(n, p+1,0)$.

But our upper bound is minimum for $p=n / 2$ which is a bit bizarre. Note that Conjecture 2 implies $R(n, p, f) \leq 18 n+34 f+O(\log ((n+f))$ for every $p$.

It seems that the cornerstone of the problem is to find the smallest constant $\alpha$ such that $R(n,\lceil n / 2\rceil, 0) \leq \alpha n$. If Conjecture 2 is true then $R(n, p, 0) \leq \alpha n$ for every $p$. Moreover, even if Conjecture 2 turns out to be false, if would imply that $R(n, p, 0) \leq 2 \alpha n$ for every $p$. Indeed, if $p \leq n / 2$, one can construct an $(n, p, 0)$-repartitor from an $(n,\lceil n / 2\rceil, 0)$-repartitor and an $(\lfloor n / 2\rfloor, p, 0)$-repartitor. (See Figure 14). Hence, $R(n, p, 0) \leq R(\lfloor n / 2\rfloor, p, 0)+R(n,\lceil n / 2\rceil, 0)$.

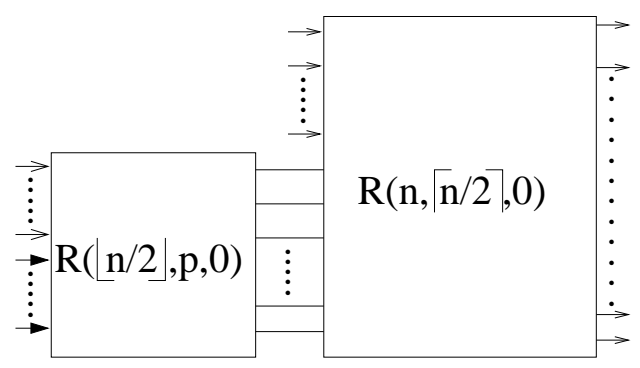

Figure 14: Construction of an $(n, p, 0)$-repartitor from an $(n,\lceil n / 2\rceil, 0)$-repartitor and an $(\lfloor n / 2\rfloor, p, 0)$-repartitor. 
Applying this formula recursively with the equality $R(n, p, 0)=R(n, n-p, 0)$, we obtain :

$$
R(n, p, 0) \leq \sum_{i=1}^{\log (n)} R\left(\left\lfloor\frac{n}{2^{i}}\right\rfloor,\left\lceil\frac{n}{2^{i+1}}\right\rceil, 0\right)
$$

A good manner to improve the upper bounds on $R(n, p, f)$ would be to improve the upper bounds for $S(p, n)$ obtained in Section 2.4. Indeed these bounds are far to be tight and a lot greater than the lower bounds given in Section 3.2 or [6]. A problem is to close the gap between the lower bounds and the upper bounds.

It would also be interesting to get some results on the shape of $S(p, n)$. We conjecture that, for any fixed integer $n, S(p, n)$ increases until an integer $\alpha_{n} \geq n / 2$ and then decreases. In particular, we conjecture :

Conjecture 3 If $p \leq n / 2$ then $S(p-1, n) \leq S(p, n)$.

If true, this conjecture would imply $S(p, n) \leq S(n / 2, n) \leq 9 n+O(\log (n))$ if $p \leq n / 2$ which is a better upper bound as ours.

One can easily prove a weaker statement than Conjecture 3 :

\section{Proposition 18}

$$
S(p-1, n-1) \leq S(p, n)
$$

Proof. The network obtained from a $(p, n)$-selector by removing an input and an output is trivially a $(p-1, n-1)$-selector.

A way to improve the upper bounds on $S(p, n)$ and so those on $R(n, p, f)$ is to improve the bounds on $S^{+}(n)$. It would also be interesting to close the gap $\frac{4}{3} n \leq S^{+}(n) \leq 17 n+$ $O(\log (n))$.

\section{References}

[1] N. Alon and M. Capalbo, Smaller explicit superconcentrators. Internet Math. 1 (2) (2003), 151-163.

[2] L. A. Bassalygo, Asymptotically optimal switching circuits, Problemy Pederachi Informatsii, 17 (1981), 81-88.

[3] B. Beauquier, and E. Darrot. Arbitrary size Waksman networks and their vulnerability. Parallel Processing Letters, 12 (3-4) (2002), 287-296.

[4] J.-C. Bermond, E. Darrot, and O. Delmas. Design of fault tolerant on-board networks in satellite. Networks, 40 (4) (2002), 202-207.

[5] J.-C. Bermond, F. Havet, and D. Tóth. Fault tolerant on-board networks with priorities. Networks, submitted. INRIA research report RR-5363.

$\mathrm{RR} \mathrm{n}^{\circ} 5686$ 
[6] J.-C. Bermond, S. Pérennes, and D. Tóth. On the design of fault tolerant flow networks, part I. Manuscript.

[7] F. R. K. Chung, On concentrators, superconcentrators, generalizers, and nonblocking networks, Bell System Tech. J., 58 (1979), 1765-1777.

[8] N. Pippenger, Superconcentrators, SIAM J. Comput., 6 (1977), 298-304. 


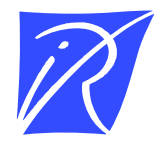

\section{Unité de recherche INRIA Sophia Antipolis 2004, route des Lucioles - BP 93 - 06902 Sophia Antipolis Cedex (France)}

Unité de recherche INRIA Futurs : Parc Club Orsay Université - ZAC des Vignes 4, rue Jacques Monod - 91893 ORSAY Cedex (France)

Unité de recherche INRIA Lorraine : LORIA, Technopôle de Nancy-Brabois - Campus scientifique 615, rue du Jardin Botanique - BP 101 - 54602 Villers-lès-Nancy Cedex (France)

Unité de recherche INRIA Rennes : IRISA, Campus universitaire de Beaulieu - 35042 Rennes Cedex (France)

Unité de recherche INRIA Rhône-Alpes : 655, avenue de l'Europe - 38334 Montbonnot Saint-Ismier (France)

Unité de recherche INRIA Rocquencourt : Domaine de Voluceau - Rocquencourt - BP 105 - 78153 Le Chesnay Cedex (France)

INRIA - Domaine de Voluceau - Rocquencourt, BP 105 - 78153 Le Chesnay Cedex (France)

http://www.inria.fr

ISSN 0249-6399 